\title{
The criterion-calibration model of cue interaction in contingency judgments
}

\author{
Samuel D. Hannah • Lorraine G. Allan
}

Published online: 10 February 2011

(C) Psychonomic Society, Inc. 2011

\begin{abstract}
Siegel, Allan, Hannah, and Crump (2009) demonstrated that cue interaction effects in human contingency judgments reflect processing that occurs after the acquisition of information. This finding is in conflict with a broad class of theories. We present a new postacquisition model, the criterion-calibration model, that describes cue interaction effects as involving shifts in a report criterion. The model accounts for the Siegel et al. data and outperforms the only other postacquisition model of cue interaction, Stout and Miller's (2007) SOCR model. We present new data from an experiment designed to evaluate a prediction of the two models regarding reciprocal cue interaction effects. The new data provide further support for the criterion-calibration model.
\end{abstract}

Keywords Contingency - Criterion setting - Criterion shift . Cue interaction $\cdot$ Signal detection theory

The study of human contingency judgments is the study of a cornerstone task in cognition. We judge how strongly contingent two events are when learning, problem solving, and engaging in almost all forms of inferential reasoning, especially causal reasoning. Contingency and causality judgments are so closely linked that research studies of them often

\footnotetext{
S. D. Hannah $(\bowtie)$

School of Psychology, University of Queensland,

St. Lucia 4072 QLD, Australia

e-mail: hannah.sam@gmail.com

L. G. Allan

Department of Psychology, Neuroscience \& Behavior,

McMaster University,

Hamilton, ON, Canada
}

share the same paradigms and are often reviewed together (e.g., Shanks, 2007). Outside the laboratory, contingency judgments are often complicated - in part, because of the many events that are co-occurring. Inside the laboratory, such complicated scenarios are often studied in a simplified analogue called the one-phase blocking task (Baker, Mercier, Valle-Tourangeau, Frank \& Pan, 1993).

In the one-phase blocking task, participants are presented with two events, often referred to as cues, that covary with another event, often described as an outcome. The target cue typically keeps a constant contingency with the outcome, with contingency usually measured by a statistic called $\Delta P=P($ Outcome $\mid$ Cue $)-P($ Outcome $\mid \sim$ Cue $)$. Like Pearson's $r, \Delta P$ varies from +1.0 for a perfect positive contingency to -1.0 for a perfect negative contingency. The target cue is paired with a companion cue that has a variable value of $\Delta P$ across conditions. The most common finding is that the judged strength of the target cue contingency varies inversely with the strength of the companion cue contingency: The stronger the companion's contingency with the outcome, the weaker the reported contingency of the target cue with the outcome. For example, participants may be required to judge the influence of a fertilizer that has a contingency of $\Delta P(.5)$ with plant growth (Spellman, 1996a). Judgments of the fertilizer's influence on plant growth are higher when a rival fertilizer has a null contingency with plant growth than when its rival fertilizer has a perfect contingency with plant growth.

Cue interaction effects like those elicited in the one-phase blocking task have been explained from several theoretical perspectives. Most associative-learning models - such as the Rescorla-Wagner model (Rescorla \& Wagner, 1972) or Pearce's (1994) configural model-interpret the results of 
the one-phase blocking task in terms of the more predictive cue's impeding an association formation process between the less predictive cue and the outcome.

For very different reasons, accounts stressing the role of reasoning also place the locus of cue interaction effects at an acquisition stage. In reasoning-based accounts, however, it is the acquisition of knowledge that changes with companion contingency, not the acquisition of associative strength. Spellman (1996a, b) conditional $\Delta P$ model, for example, describes cue interaction effects in one-phase blocking experiments as the result of participants' taking into account only those target events that occur with the companion always present or always absent. Spellman has argued that participants make their decisions regarding the target conditional on the companion's presence or absence.

In contrast to these acquisition accounts of cue interaction effects, Miller's comparator hypothesis (e.g., Miller \& Matzel, 1988) and its mathematical formulation, sometimes-competing retrieval (SOCR) by Stout and Miller (2007), places the locus of cue interaction effects at test, after cues have formed an association with the outcome. According to Miller, the strength of responding to a cue reflects the cue's associative strength relative to that of its rivals, where the rivals are retrieved from memory. As rival cues gain in associative strength, responding to a target cue weakens. In a one-phase blocking task, therefore, ratings of a constant target contingency must drop as companion contingencies increase in strength. Miller and colleagues have shown a number of counterintuitive results that flow from this hypothesis, such as that responding to a conditioned cue changes with posttraining treatment to its companion cues even though no further information about the conditioned cue has been presented (e.g., Blaisdell, Bristol, Gunther \& Miller, 1998; Denniston, Savastano, Blaisdell \& Miller, 2003).

Although we described SOCR as the only postacquisition model of cue interaction effects, there are other models of learning or conditioning that also include a postacquisition component. Pineño (2007), for example, offered a variant on SOCR's (Stout \& Miller, 2007) response rule wedded to a competitive learning mechanism, allowing Pineño to account for cue interaction as a postacquisition phenomenon, as Stout and Miller do, or to treat it as arising from competition in acquisition, as most associativelearning models do. Gallistel and Gibbon's (2000) RET model describes a conditioned response as occurring when a signal based on reinforcement intervals exceeds a decision threshold. However, they explicitly described cue interaction as arising during signal acquisition, from a mechanism that attributes reinforcement signals to different cues. RET has the tools to describe cue interactions as postacquisition effects, but it locates them squarely in the acquisition process.
We thus have a large group of theories that locate cue interaction effects in the acquisition of a signal, regardless of whether the signal is understood as consisting of associative strength or propositional information. A much smaller group of theories, consisting of just one theory, locates cue interaction effects after signal acquisition, at a comparison stage immediately prior to responding. If we had a way of distinguishing the influence of acquisition from any later process, we could distinguish between these camps. Fortunately, signal detection theory (Green \& Swets, 1966) provides just such tools.

Over the next sections, we will outline how signal detection theory has been applied by Siegel et al. (2009) to the study of cue interaction effects by blending a novel technique, Crump's streamed-trial procedure (Crump, Hannah, Allan \&, Hord 2007), with an old psychophysical technique, the method of constant stimuli. This merging of methods produces results consistent with a postacquisition account of cue interaction. We will describe a new postacquisition model, and we will demonstrate that our criterion-calibration model accounts well for Siegel et al.'s data, even better than Stout and Miller's (2007) formalization of the comparator hypothesis, known as SOCR. We will also show that the criterion-calibration model successfully predicts a novel finding that conflicts with the predictions of SOCR.

\section{Signal detection theory and the streamed-trial procedure}

Signal detection theory decomposes responding into a sensitivity component and a report criterion. Sensitivity reflects the efficiency of signal acquisition, while a report criterion allows an acquired signal to be interpreted by comparison with a criterion. To apply signal detection theory, researchers must be able to measure responding to the signal across trials. In a conventional contingency or causality judgment task, however, a contingency is a signal that accumulates over a block of trials. To ensure a triallevel response to a contingency value, a method has to be found to turn the block of trials into a single trial.

Crump's development of the streamed-trial procedure (Crump et al., 2007) performs such a transformation by presenting cue and outcome simultaneously and rapidly $(100 \mathrm{~ms})$. Figure 1 gives schematics for streamed trials in both single-cue (panel A) and one-phase blocking (panel B) paradigms. In the single cue-task that Crump et al. used, the cue was the square (blue in the experiment), and the outcome was the circle (red in the experiment). In Hannah, Crump, Allan, and Siegel's (2009) use of the streamed-trial procedure in a one-phase blocking task, the target cue could be either the square or the triangle (both blue in the experiment), and 

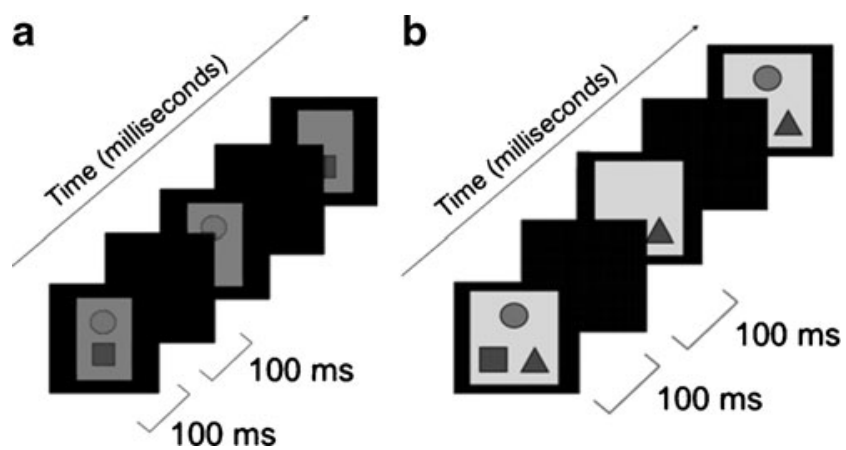

Fig. 1 Schematic of streamed-trial procedure used a in Allan et al. (2008) and b in Hannah et al. (2009), and Siegel et al. (2009)

the outcome was again the red circle. Whichever cue was the target, its contingency with the outcome always had a $\Delta P$ value of .5 , or $\Delta P(.5)$, while the companion's appearance was either perfectly contingent with the circle or noncontingent.

Despite the rapid display and simultaneous presentation of cues and outcomes, Crump and colleagues showed that the streamed-trial procedure elicits the benchmark results of traditional contingency tasks. The procedure elicits systematic biasing of contingency ratings by outcome frequency (Crump et al., 2007), and cue interaction effects as found in one-phase blocking and two-phase blocking (Hannah et al., 2009) tasks, including both forward and backward blocking.

\section{Psychophysics and cue interaction effects}

Allan, Hannah, Siegel, and Crump (2008) combined the streamed-trial procedure with a psychophysical technique often used with signal detection theory. An experimenter using the method of constant stimuli presents different signal strengths, and the participant makes a binary response-for example, present/absent, strong/weak. By varying the strength of the signal, the experimenter can track the relation between responding and signal strength, tracing out a curve called a psychometric function. An idealized example of a psychometric function is shown in Fig. 2. As stimulus strength increases along the $x$-axis, the increase in the probability of making one of the responses (y-axis) follows a sigmoid function. When the method of constant stimuli is used to track responding in a one-phase blocking task, signal strength is the level of $\Delta P$ for either the target or the companion cue, and the tracked response is the probability of identifying a cue's contingency as strong.

The slope of a psychometric function is an index of sensitivity when fitted by a signal detection model, given some standard assumptions (see Allan et al., 2008; Killeen, Fetterman \& Bizo, 1997). The steeper the slope, the more sensitive is signal acquisition. Therefore, if two conditions produce differences in behavior due to differences in acquiring information, the two conditions will yield functions that differ in slope.

The psychometric function also provides a simple way of assessing report criterion. Report criterion is the border between the two binary responses, and therefore, it is the point at which each response is equally probable. The value of the report criterion is the signal strength that corresponds to a $50 \%$ response probability. Visually, it is the point on the $x$-axis at which the psychometric function reaches the $50 \%$ mark on the $y$-axis. This point is called the point of subjective equality (PSE). In Fig. 2, this is just past the fourth strength level. If two conditions produce differences in behavior due to differences in decision after information acquisition, the two conditions will yield functions that differ in PSE.

To apply the method of constant stimuli to one-phase blocking, a researcher can use typical contingency values for the companion cues- $\Delta P(0.0)$ and $\Delta P(1.0)$-and can vary the strength of the target cue. For each companion, there would be a range of target cue strengths, resulting in two target functions, one describing responding to targets in the presence of a noncontingent companion (a target function) $^{2}$ and one describing responding to targets in the presence of

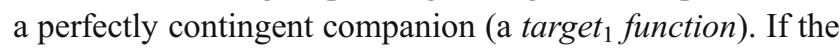
strength of the companion contingency changes what information is extracted from the display, the slopes of the two target functions will differ. If, however, the presence of the companion changes only the interpretation of the target contingency, the two functions will have the same slope but different PSEs.

\section{Psychophysics, cue interactions, and streamed trials}

Recently, Siegel et al. (2009) combined the method of constant stimuli with Hannah et al.'s (2009) streamed-trial

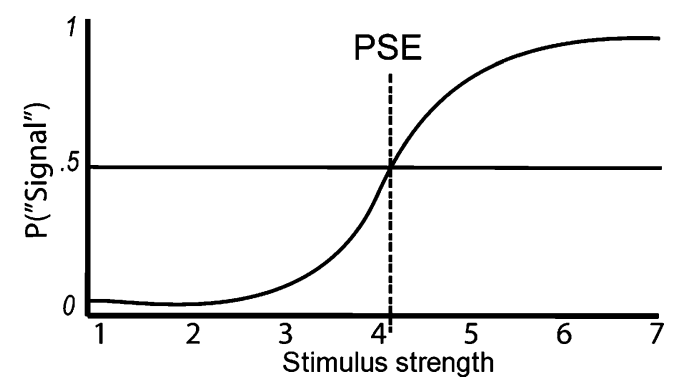

Fig. 2 Idealized psychometric function. The function plots $P$ ("Signal") against increasing levels of stimulus strength. The value on the $x$-axis (dotted line) at which $P$ ("Signal") $=.5$ is the point of subjective equality (PSE), which corresponds to the location of the report criterion 
adaptation of the one-phase blocking task. Participants in Siegel et al.'s task were given streamed trials on which each 100-ms stimulus display, or frame, consisted of some combination of an outcome, target cue, and companion cue. A trial schematic is given in Fig. 1b. Cues consisted of squares and triangles, and the outcome was a circle. At the end of each streamed trial, a small picture of the circle and the square or of the circle and the triangle appeared. Participants judged whether the contingency indicated by the picture was strong or weak and made their report by clicking on a button labeled "Strong" or on a button labeled "Weak."

For any trial, the companion cue had a contingency with the outcome of either $\Delta P(0.0)$ or $\Delta P(1.0)$ (companion $_{0}$ and companion $_{1}$ streams, respectively), and the target cue had a contingency ranging from $\Delta P(.2)$ to $\Delta P(.8)$ in increments of $\Delta P(.2)$. Actual frequencies of different frames for each companion $\times$ target trial type are given in Tables 1 (companion $_{0}$ streams) and 2 (companion ${ }_{1}$ streams). The eight different types of streamed trials created by the 2 (companion $\Delta P) \times 4($ target $\Delta P)$ contingency levels were presented 30 times each in every hour-long session, with each participant running in 15 sessions. Siegel et al. (2009)

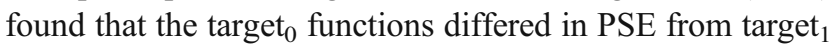
functions but did not differ in slope. Siegel et al. concluded that the cue interaction effect in their data reflected changes in report criterion, not sensitivity. Cue interaction effects, therefore, reflect the operation of mechanisms occurring after signal acquisition, at some comparison stage, supporting accounts such as Miller's comparator hypothesis (Miller \& Matzel, 1988; Stout \& Miller, 2007). Laux, Goedert, and Markman (2010) recently examined causality judgments in a one-phase blocking task, using the streamed-trial procedure, and also found only report criterion differences.

The target cue functions allowed Siegel et al., (2009) to see whether the value of the companion contingency changed the slope (sensitivity) or PSE (criterion) of target cue functions. The companion cue functions, however, allowed them to do something just as novel. In the

Table 1 Four contingency matrices defining the different stream

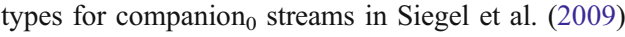

\begin{tabular}{|c|c|c|c|c|c|c|c|c|}
\hline & \multicolumn{8}{|c|}{ Target Cue Contingency } \\
\hline & \multicolumn{2}{|l|}{.2} & \multicolumn{2}{|l|}{.4} & \multicolumn{2}{|l|}{.6} & \multicolumn{2}{|l|}{.8} \\
\hline & $O$ & $\sim O$ & $O$ & $\sim O$ & $O$ & $\sim O$ & $O$ & $\sim O$ \\
\hline$A B$ & 8 & 6 & 9 & 5 & 10 & 4 & 10 & 2 \\
\hline$A \sim B$ & 2 & 4 & 1 & 5 & 0 & 6 & 0 & 8 \\
\hline$\sim A B$ & 4 & 2 & 5 & 1 & 6 & 0 & 8 & 0 \\
\hline$\sim A \sim B$ & 6 & 8 & 5 & 9 & 4 & 10 & 2 & 10 \\
\hline
\end{tabular}

$A$ denotes the companion cue, and $B$ denotes the target cue
Table 2 Four contingency matrices defining the different stream types for companion ${ }_{1}$ streams in Siegel et al. (2009)

\begin{tabular}{|c|c|c|c|c|c|c|c|c|}
\hline & \multicolumn{8}{|c|}{ Target Cue Contingency } \\
\hline & \multicolumn{2}{|l|}{.2} & \multicolumn{2}{|l|}{.4} & \multicolumn{2}{|l|}{.6} & \multicolumn{2}{|l|}{.8} \\
\hline & $O$ & $\sim O$ & $O$ & $\sim O$ & $O$ & $\sim O$ & $O$ & $\sim O$ \\
\hline$A B$ & 12 & 0 & 14 & 0 & 16 & 0 & 18 & 0 \\
\hline$A \sim B$ & 8 & 0 & 6 & 0 & 4 & 0 & 2 & 0 \\
\hline$\sim A B$ & 0 & 8 & 0 & 6 & 0 & 4 & 0 & 2 \\
\hline$\sim A \sim B$ & 0 & 12 & 0 & 14 & 0 & 16 & 0 & 18 \\
\hline
\end{tabular}

$A$ denotes the companion cue, and $B$ denotes the target cue

conventional one-phase blocking task, the target cue is defined as the cue that is constant across conditions. Since the target contingency is not varied in typical implementations of the one-phase blocking task, the influence of the target cue on the companion cannot be measured, and any potential reciprocal cue interaction effects cannot be examined. In the psychophysical implementation of one-phase blocking, however, the target cue necessarily must vary, providing the potential to observe reciprocity in cue interaction effects. Such reciprocal effects were the focus of Experiment 1, which we will present in more detail later.

The signal detection analysis Siegel et al., (2009) applied to their data indicated that the cue interaction effect was attributable to the location of the criterion shifting with the value of the companion contingency. However, Siegel et al. did not present a process describing how this criterion shift occurs. In the next section, we introduce a new model, the criterion-calibration model of cue interaction effects. Like the comparator hypothesis of Miller and colleagues, recently formalized as the SOCR model by Stout and Miller (2007), the criterion-calibration model is a postacquisition model. In the next section, we introduce the new model and then fit it and SOCR to Siegel et al.'s data. We then present a new experiment designed to differentiate between the two models.

\section{The criterion-calibration model of cue interaction effects}

Signal detection theory: A beginning

Signal detection theory treats responding as an assessment of events that vary along an internal dimension of strength or evidence. In the simplest scenario, the events are a mixture of meaningful signals and irrelevant noise. In a loudness judgement, for example, the events might be loud versus soft tones. However, the events come with no tags to 
identify them, only different levels of subjective strength that are typically treated as being normally distributed over trials. Because these distributions are usually expected to be overlapping, some signals from a usually strong signal source may sometimes be perceived as no stronger than most signals from a usually weak signal source, and vice versa. Decision making cannot be supported by subjective strength alone. Signal detection theory posits, therefore, that people interpret these values relative to a report criterion. It is this report criterion that is used to decide how loud is "loud," or how strong is "strong.".

While the transduction of signals is considered to be fairly stable and robust, the report criterion (more simply, criterion) is considered to be more plastic, influenced by expectations, by the perceived costs and benefits of different types of errors and successes, and by many other cognitive factors. However, once a report criterion is established within a task, it is usually treated as fixed until the experimental conditions change, such as a change in reward levels or a change in block instructions. ${ }^{1}$

What is a rational or ideal position for a criterion? In Siegel et al.'s (2009) paradigm, there are equal costs and benefits to each decision, a simple binary response to be made, and no reason to expect one event to be more likely than its complement. Under these conditions, we suggest that the least arbitrary place for a criterion is in the middle of the range of experienced events, so that half are called strong and half are called weak; where there is no environmental bias, a rational observer should attempt to be unbiased in their criterion setting. ${ }^{2}$ However, the range is being defined as an experiment unfolds. The dynamic nature of experiments poses a challenge to the observer trying to maintain a mid-range criterion, and this challenge is where our account begins.

The criterion-calibration model: Basic assumptions

As the middle of a range of values is difficult to identify while the range unfolds, we expect that people infer its

\footnotetext{
${ }^{1}$ The importance of trial-level criterion variability has been highlighted in some recent accounts (e.g., Benjamin, Diaz, \& Wee, 2009) and has been incorporated into many approaches in the recognition memory literature (see Stretch \& Wixted, 1998). However, few accounts have formalized the process of criterion shifting. The relation of the criterion-calibration model to the models that do describe criterion shifting will be developed in the General Discussion section, along with examinations of our work in relation to other treatments of criterion variance.

${ }^{2}$ Although we are assuming that the observers are rational, we are not assuming that they are ideal observers (e.g., Green \& Swets, 1966). That is, we are not assuming that they are making use of all the information to make an optimal decision. Indeed, given the ambiguities inherent in a judgment like "strong" or "weak," it is not immediately obvious what an optimal decision should be.
}

location by assuming that if the criterion is in the middle of the range, most contingencies should be near the criterion. When trying to maintain the criterion in the middle of the range, far contingencies - that is, contingencies that are far above or far below the current criterion-provide evidence for the criterion being in the wrong place and needing calibration. How willing a person is to interpret the distance between a contingency and criterion as evidence for adjustment is likely to vary with individuals and tasks and is a parameter in the model. The more confident a person is that the distance an event falls from the current criterion is evidence of criterion "error," the larger the shift they should make in response to a far contingency.

An experienced contingency that is far from the criterion is evidence of an error that is to be reduced, and the participant reduces criterion error by moving the criterion toward the contingency value. This shift, however, must be weighted by the distance between contingency and criterion, since only far contingencies are evidence of criterion error; near contingencies confirm the criterion's location. We could try to construct a criterion shift function with a sharp threshold, or even a bivalent function that entrenches the criterion for near contingencies while moving it for far contingencies. It seemed simpler, however, to seek a continuous function that allows a selective, nonlinear weighting of contingency - criterion (Cont - Crit) differences, muting shift size for near contingencies,and sharply increasing the shift size for far contingencies. Of course, the increase in shift size with far events must asymptote at some point, or responding would become catastrophically unstable.

Why the hyperbolic tangent (cubed)? We wanted a criterion shift function that adjusts the criterion by the difference between a contingency's subjective strength and the current criterion (Cont - Crit), but one that (1) weights this difference selectively, so that far contingencies produce a big shift but near contingencies yield a negligible shift, (2) preserves the sign of the Cont - Crit difference because the direction of shift is meaningful, and (3) has an asymptote. Thus, the function must get increasingly negative for negative differences and increasingly positive for positive differences, produce no shift for zero difference (i.e., be centered on zero), and be bounded. What is called for is a bounded, or clamped, exponential-like function-that is, a sigmoid function. This suggests the following:

$\frac{e^{x}-e^{-x}}{e^{x}+e^{-x}}$

where $x$ is the Cont - Crit difference. When $x$ is zero, Eq. 1 reduces to $(1-1) / 2=0$, producing a zero shift given zero 
difference. As $x$ (the Cont - Crit difference) gets very large, the term $e^{-x}$ in both the numerator and denominator approaches zero, and the function approaches $e^{x} / e^{x}= \pm 1$, depending on the sign of $x$.

Equation 1 can be more simply expressed as the hyperbolic tangent (tanh) function. However, as is shown in Fig. 3, the tanh function (dotted line) weights large values only slightly more than it does small values. Evenvalued exponents, however, produce only positively valued outputs. Thus, a cubic exponent (Fig. 3, solid line) represents the smallest integer-valued exponent that preserves the sign of Cont - Crit and also yields substantial selectivity in weighting Cont - Crit. The output of the cubed tanh function is multiplied by a participant-specific factor that captures individual differences across conditions. We call this participant-specific scale factor the shift adjustment factor (SAF) and take it as reflecting the willingness to treat the distance between an event and the criterion as evidence of criterion error.

The cubed tanh function can be shown to be inevitably pulled to the middle of the range, at least in principle, given an equal presentation of range values. ${ }^{3}$ Other sigmoidal functions are available, but they are less convenient to implement, and it is unclear whether they result in the criterion's converging to the middle of the range. What is important for this article, however, is establishing the validity of the ideas that our shift function instantiates, not the specifics of the implementation.

In Fig. 4, we illustrate how this scaled, nonlinear shift function adjusts the criterion across two streamed trials, a companion $_{0}$ stream (top panels) and a companion $_{1}$ stream (bottom panels). For simplicity, we assume that the participant first evaluates the stronger of the two contingencies on each streamed trial, with the events treated as representations of some subjective strength based on a noisy transduction of objective contingency. In this example, we also assume that the participant begins with a criterion placed below the middle of the $\Delta P$ range and that the target cue contingencies ( $T 1$ and $T 2$ ) produce identical representations on the two trials.

On the first streamed trial (top panels in Fig. 4), the participant evaluates the target-outcome contingency first; the representation of this contingency, $T 1$, is a short distance above the criterion. The participant makes a "strong" decision, ${ }^{4}$ and the criterion is shifted by a negligible amount toward $T 1$ (top left panel). The size of this shift is determined both by the shift function and by

\footnotetext{
${ }_{3}^{3}$ For those interested, a demonstration of convergence is available from the first author.

${ }^{4}$ If this were a Siegel et al. (2009) type experiment, the participant would not necessarily report this decision, unless it was the probed contingency.
}

the participant-specific SAF, which, in principle, can range from $-\infty$ to $\infty$ but seems usually to range between 0 and 4 , on the basis of our data fitting. We set the participant's SAF to 1.5. The shift function mutes differences of less than 0.5 units to almost nothing, and almost nothing multiplied by 1.5 is still almost nothing. The shift in criterion in response to the first targetoutcome contingency is negligible.

Next (top right panel in Fig. 4), the contingency of the companion $_{0}$ cue with the outcome $(C 1)$ is evaluated. This contingency is further away from criterion than the targetoutcome contingency was and is below the criterion. The participant classifies the contingency as "weak," and the function produces a shift slightly larger than the $T 1$ shift. This modest output from the shift function is modestly enlarged by the participant's SAF. A small but observable shift toward the companion contingency occurs; the participant becomes slightly more liberal in his or her responding.

On the second streamed trial (bottom panels in Fig. 4), the companion contingency is stronger than the target contingency, and thus the participant first evaluates the companion $_{1}$ cue's contingency (C2), making a "strong" decision. $C 2$ is far from the current criterion position, and the difference between the criterion and companion contingency generates a large output from the shift function, multiplied further by the participant's SAF of 1.5. The resulting shift is so large that the criterion is pulled above the contingency for the target cue (T2). Thus, even though $T 1$ and $T 2$ have identical values, $T 2$ is judged to be a weak contingency, but $T 1$ is judged to be a strong contingency, producing the companion-influenced evaluation of target contingency that is the cue interaction effect.

\section{Criterion-calibration model of cue interaction effects:} Details

The model simulates trial-by-trial responding of participants; simulated responses are aggregated for each run of the model, and comparison with actual data is based on the aggregate percentage of trials called "strong." Each simulated decision in the model consist of four steps - signal transduction, signal evaluation, decision, and criterion adjustment - the first three being standard operations in a signal detection framework. Model parameters are summarized in Table 3, and the implementation code and pseudocode are available from the first author.

The four steps of a trial decision are serially applied to each of the two cues in the one-phase blocking task. We make no assumptions as to how stimuli are transduced to internal values. We are content to describe internal values of signal strength as behaving as if drawn from a normal distribution with a mean approximated by the $\Delta P$ value of 
Fig. 3 Plot of the function $y=\tanh (x)$ (dotted line) and the function $y=\tanh (x)^{3}$ (solid line), where $x=$ subjective contingency - criterion. The latter function is the basis of the criterion shift function used in the criterion-calibration model

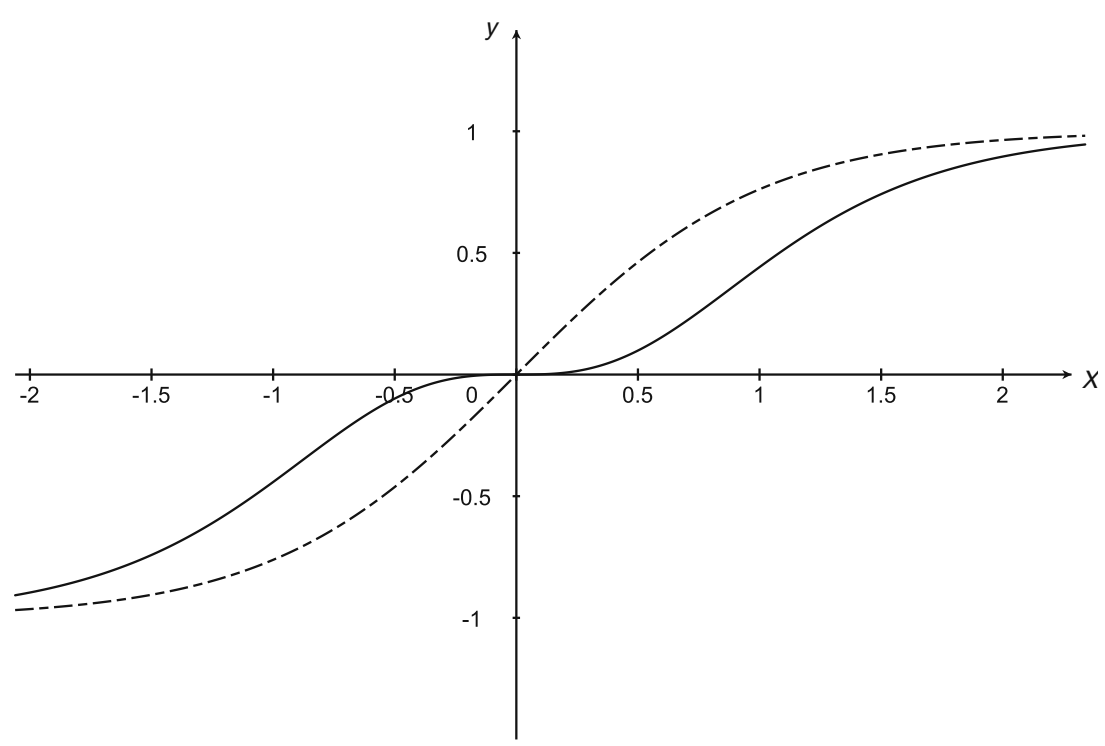

the evaluated cue $\mathrm{e}^{5}$ and with a standard deviation that is a participant-specific parameter $(\sigma)$ estimated from each participant's data; this $\sigma$ parameter drives sensitivity effects in the criterion-calibration model. That there is only a single $\sigma$ parameter per participant implies that sensitivity is constant across target functions for any participant, regardless of companion contingency.

In the model, a response is determined by subtracting the position of the report criterion from the value of the cue on the subjective strength dimension. If this difference is negative, the model makes a "weak" response; if positive, it makes a "strong" response. After the model makes its response, the criterion is adjusted by adding the difference between the event and criterion back to the criterion, after transforming the difference with the cubed hyperbolic tangent shift function:

Criterion Position $_{i+1}={\text { Criterion } \text { Position }_{i}}$

$$
+\tanh \left(\text { Cont }_{i}-\text { Crit }_{i}\right)^{3}
$$

This shift is scaled by the participant's SAF. Thus, the final form of the model's criterion-shift function is

$$
\begin{aligned}
\text { Criterion Position }_{i+1}= & \text { Criterion Position }_{i} \\
& +\left(S A F \times \tanh \left(\text { Cont }_{i}-\text { Crit }_{i}\right)^{3}\right)
\end{aligned}
$$

\footnotetext{
${ }^{5}$ Like Allan et al. (2008), we used the simplest signal detection model. We assumed that perceived contingency increased linearly with $\Delta P$ and that the variability in perceived contingency had a Gaussian distribution with a constant variance.
}

There are at least two different assumptions we can make about the constancy of the SAF value. The simplest is that the SAF is constant for all conditions in the experiment. However, the SAF reflects the participants' confidence in treating the difference between a cue and the criterion as meaningful evidence. Such confidence could be influenced by confidence in their own performance; we might, therefore, assume that the size of the SAF varies with task difficulty. Pilot work in our laboratory comparing the discrimination of different pairs of contingencies found that the discriminability for comparisons involving $\triangle P(1.0)$ versus $\Delta P(.8)$ is higher than for comparisons involving $\Delta P$ (.6) versus $\Delta P(.4)$ or $\Delta P(.2)$ versus $\Delta P(0.0)$. Thus, companion contingencies in companion ${ }_{1}$ streams may be more discriminable than companion contingencies in the

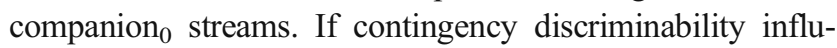
ences SAF size, SAF size may differ across companion ${ }_{1}$ and companion $_{0}$ streams. We used a model that has separate SAF parameters for each companion condition $\left(\mathrm{SAF}_{0}, \mathrm{SAF}_{1}\right)$; if there is really only a constant SAF, both SAF values should be very similar. Therefore, including the estimate of $\sigma$, the model has a maximum of three free parameters.

The criterion-calibration model assumes serial evaluation of cues, with cues altering the evaluative context for cues following them. ${ }^{6}$ The assumption of serial evaluation means that the order of cue evaluation is important. We considered two plausible strategies for choosing evaluation order: (1)

\footnotetext{
${ }^{6}$ This assumption has been supported by debriefings of participants across several streamed-trial implementations of one-phase blocking, including Siegel et al.'s (2009) participants. Although only one cue is probed on each trial, participants do not know which cue will be probed until the end of the trial; thus, the uncertainty of report reasonably forces participants to assess both cues.
} 
Fig. 4 Illustration of criterion shifting in the criterioncalibration model. The solid vertical lines indicate the position of the report criterion prior to evaluating a contingency, and the dashed vertical line indicates its shifted position after evaluating the contingency. Arrows indicate the direction of shift. Two types of stimulus events are shown: companion contingencies ("C1" and "C2") and target contingencies ("T1" and "T2")
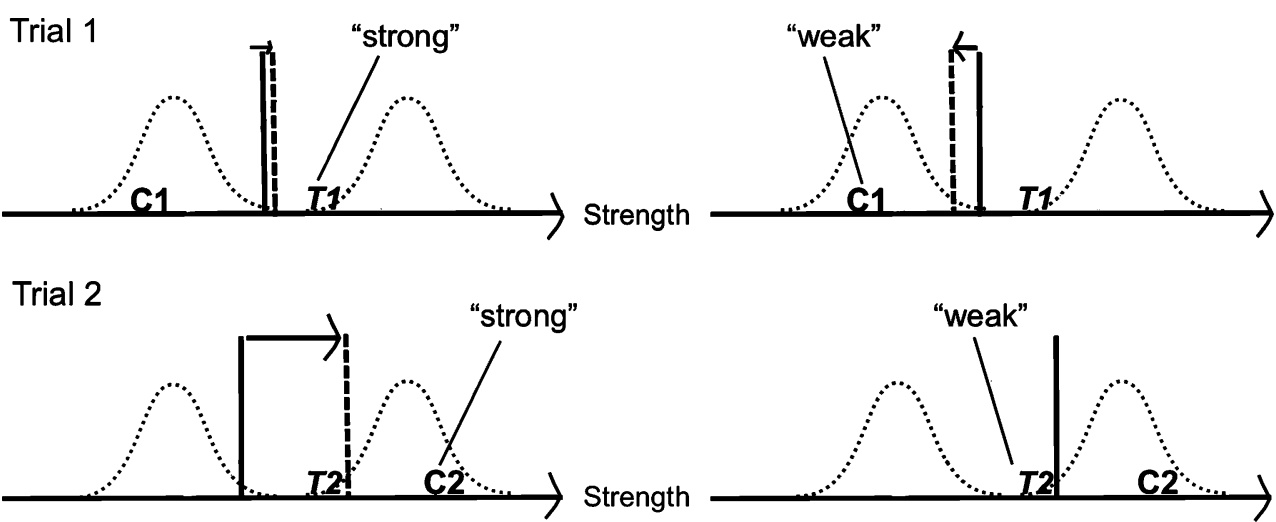

Strength

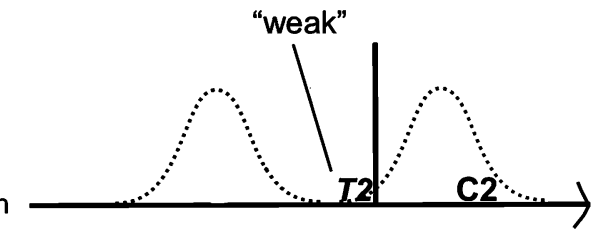

evaluate the strongest, and most obvious, contingency first (fixed evaluation strategy) $^{7}$ or (2) evaluate contingencies randomly (random evaluation strategy). Both of these strategies are built into the model. When the difference in the strength of cues is substantial enough for it to be obvious which cue is the stronger, the stronger cue's contingency is evaluated first. Otherwise, the cues are randomly chosen for evaluation. A difference in event strength is considered as "obvious enough" if it is equal to or greater than twice the simulated participant's $\sigma$ value (Strength Event $1_{1}-$ Strength $_{\text {Event } 2} \geq 2 \sigma$ ).

Such a threshold represents a conservative approach to defining obvious. To be an obvious difference, a difference should be large enough to be rarely mistaken. For the weaker cue to be mistaken for the stronger with a $2 \sigma$ threshold, not only would the weaker cue have to be stronger than the actual stronger cue by two standard deviations, but at least one cue's representation would likely have to be several standard deviations removed from its mean. Therefore, such confusion would be rare, unless the person had a very large value of $\sigma$ implying in itself that he or she has troubling distinguishing contingencies. ${ }^{8}$

\footnotetext{
${ }_{7}$ It may seem strange to talk about evaluating a contingency before it has been evaluated relative to a criterion, but the criterion is necessary only to interpret events with regard to some response scheme; hence, it is a report criterion. Assessing the ordinal strengths of events does not require interpretation with regard to any external scale.

${ }^{8}$ For example, suppose that a person had a sigma value of 0.2 and that, for ease of exposition, objective $\Delta P$ values are mapped directly onto to some subjective representation of contingency. Imagine a trial on which the target had a contingency of $\Delta P(.8)$ and the companion had a contingency of $\Delta P(1.0)$ - one of the two most likely cases in which the threshold fails to distinguish the correct ordering of objective events - and suppose that the target was transduced to a value close to that of the mean of the companion, 1.0, or one standard deviation above its objective value of .8. Although this would happen roughly $25 \%$ of the time, the weaker contingency would be taken as the stronger only if the perfect contingency were transduced to a 6 , two standard deviations below the companion's mean. Not only would this happen on about $5 \%$ of the trials, the joint probability of both happening is roughly $1 \%$. If the threshold is inaccurate, of course, it will distort what people actually do and should impair the model's fit.
}

Within the model, therefore, differences in sensitivity are linked to differences in evaluation strategies. A simulated participant with a low value of $\sigma$ will rely largely on a fixed evaluation strategy, whereas a simulated participant with a high value of $\sigma$ will rely largely on a random evaluation strategy. Thus, while the cue interaction effect in our model is immediately produced by criterion shifts, some aspects of criterion shifting are influenced by sensitivity. ${ }^{9}$

\section{The SOCR model}

We will provide only a brief description of the SOCR model (Stout \& Miller, 2007), since its details are already published. Model parameters are summarized in Table 4, and implementation code and pseudocode are available from the first author. SOCR describes learning as a noncompetitive associative process: Cue interactions such as those observed in one-phase blocking arise as a result of competition for the control of responding at test. Responding reflects not some absolute level of associative strength of a pair of stimuli, but their relative strength. More precisely, SOCR describes the strength of responding $(R)$ to a cue $(X)$ that has been associated with an outcome $(O)$ as a function of its associative strength with the outcome $\left(V_{X O}\right)$ minus the product of the associative strength of rival cues with the outcome $\left(V_{A O}\right)$ and the associative strength of $X$ with each rival cue $\left(V_{X A}\right)$. For empirical reasons, the product involving the rival cues is constrained to a

\footnotetext{
${ }^{9}$ The cubed tanh function has not played a prominent role in the psychological literature, and there might be other mathematical functions that would do as well. The cubed tanh function was chosen because, as a steeply sloped sigmoidal function, it produces substantial shifts only to events far from criterion, thus instantiating the idea that people use far deviations from a criterion as evidence of error in criterion placement. Any substitute function involving a steeply sloped sigmoidal form or some other means of implementing the idea of a selective shift in criterion toward far signals would be instantiating the same theoretical underpinnings as the present model.
} 
Table 3 Summary of parameters for criterion-calibration and SOCR models

\begin{tabular}{|c|c|c|}
\hline Model & Parameter & Function \\
\hline \multirow[t]{3}{*}{$\begin{array}{l}\text { Criterion } \\
\text { calibration }\end{array}$} & $\mathrm{SAF}_{0}$ & $\begin{array}{l}\text { Controls size of shift for a given } \\
\text { discrepancy between signal and } \\
\text { criterion in companion } \text { streams }^{-}\end{array}$ \\
\hline & $\mathrm{SAF}_{1}$ & $\begin{array}{l}\text { Controls size of shift for a given } \\
\text { discrepancy between signal and } \\
\text { criterion in companion } \text { streams }_{1} \text { strion }\end{array}$ \\
\hline & $\sigma$ & $\begin{array}{l}\text { The standard deviation of the } \\
\text { distributions for the subjective } \\
\text { signals corresponding to objective } \\
\text { contingencies; the subjective } \\
\text { variability in processing } \\
\text { contingencies }\end{array}$ \\
\hline \multirow[t]{5}{*}{ SOCR } & $k 1$ & $\begin{array}{l}\text { Controls the extent to which the } \\
\text { pairing of a cue with an absent } \\
\text { outcome or cue will weaken the } \\
\text { strength of association for the } \\
\text { cue/outcome or cue/cue pair }\end{array}$ \\
\hline & $k 2$ & $\begin{array}{l}\text { Controls the extent to which rival } \\
\text { cue associations weaken } \\
\text { responding to a focal cue }\end{array}$ \\
\hline & $s 1$ & $\begin{array}{l}\text { Saliency parameter for companions; } \\
\text { controls the influence of companion } \\
\text { stimuli on association formation }\end{array}$ \\
\hline & $s 2$ & $\begin{array}{l}\text { Saliency parameter for outcomes; } \\
\text { controls the influence of outcome } \\
\text { stimuli on association formation }\end{array}$ \\
\hline & $s 3$ & $\begin{array}{l}\text { Saliency parameter for targets; } \\
\text { controls the influence of target } \\
\text { stimuli on association formation }\end{array}$ \\
\hline
\end{tabular}

maximum value of 1.0 and is multiplied by a competition parameter, $k 2$, yielding

$R_{X}=V_{X O}-k 2\left(\sum V_{X A} \times V_{A O}\right)$

Calculating the associative strength terms $\left(V_{X O}, V_{X A}, V_{A O}\right)$ requires a saliency parameter for each relevant stimulus present in a given display. Applying the model to Siegel et al. (2009) data requires a separate saliency parameter for each of the target, companion, and outcome. When one stimulus is present and another not, the associative strength term for that pair is decreased by a general decrement parameter, $k 1$. Finally, the formula given in Eq. 4 excludes a term called the switching operator that is needed to give the SOCR model the capacity to account not only for the competitive effects of multiple cues, but also for the findings that, sometimes, rival cues are facilitative. The switching operator normally requires a sixth parameter, called $k 3$. However, we are considering a restricted situation, that of cue competition alone, a situation for which the switching parameter quickly asymptotes at 1.0. We therefore fixed the switching parameter to 1.0 , reducing the number of free parameters in our implementation of the model.

Finally, we note that $R_{X}$ indexes the strength of responding to a cue versus not responding to the cue. We take this as a binary response situation analogous to Respond"Strong" versus Respond "Weak", given that the experimental situation does not allow for not responding. Thus, $R_{X}$ in our model indexes the strength (treated as a response probability) of making a "strong" response; the probability of making a "weak" response is $1-R_{X}$, making it a default response, since the model responds "weak" unless sufficient associative strength builds up.

\section{Simulation results and discussion}

When fit to Siegel et al. (2009) data, the SOCR model requires five parameters that must be estimated from the data-three saliency parameters $(s 1, s 2, s 3$, confined to the interval 0 and 1 , inclusive) and two general responding parameters $(k 1, k 2)$-whereas the criterion-calibration model requires three $\left(\sigma, \mathrm{SAF}_{0}, \mathrm{SAF}_{1}\right)$. The order of cue evaluation is not a free parameter, since it depends on $\sigma$.

We used a Nelder-Mead simplex algorithm (Nelder \& Mead, 1965) to fit the data and estimate parameters for both models. The simplex is a set of points in a multidimensional space, whose dimensionality is equal to the number of model parameters plus one $(n+1)$. When the function to be minimized is the root-mean squared deviation (RMSD)between a model and a set of data, the algorithm usually finds parameters that yield the best fit. To begin, the model is fit $n+1$ times, and for each of these initial fits, one parameter is changed and the value of the function (RMSD in our case) is calculated. For the last fit, only the starting (unchanged values) are used. The simplex is thus a set of $n+1$ configurations of parameter values.

Table 4 Estimated parameter values and fit indices for the criterioncalibration model, all participants, Siegel et al. (2009)

\begin{tabular}{|c|c|c|c|c|c|}
\hline \multirow[b]{2}{*}{ Participant } & \multicolumn{3}{|c|}{ Parameters } & \multirow[b]{2}{*}{$R M S D$} & \multirow[b]{2}{*}{$R^{2}$} \\
\hline & $\mathrm{SAF}_{0}$ & $\mathrm{SAF}_{1}$ & $\sigma$ & & \\
\hline X.G. & 2.75 & 3.66 & 0.13 & 0.052 & .982 \\
\hline M.C. & 1.58 & 3.04 & 0.12 & 0.030 & .994 \\
\hline A.S. & 0.01 & -0.01 & 0.48 & 0.044 & .961 \\
\hline A.B. & 2.13 & 0.54 & 0.41 & 0.064 & .945 \\
\hline S.A. & 0.20 & 2.80 & 0.18 & 0.022 & .996 \\
\hline C.H. & -0.40 & 2.32 & 0.22 & 0.045 & .973 \\
\hline
\end{tabular}

"SAF 0 " indicates the SAF parameter for companion streams. "SAF $_{1}$ " indicates the SAF parameter for companion $_{1}$ streams. Actual and simulated data are shown in Fig. 5 
After this initial round of fitting, the simplex is reflected along an axis defined by the centroid (mean) of the simplex and the best-fitting point. If this produces a new point that fits better than the remaining points, the worst-fitting point is replaced by extending the original reflection. Otherwise, the simplex is contracted along the line of reflection. By successive reflections, expansions, and contractions, the parameter space is explored, and the values that minimize the function are usually found. Initialization and fitting algorithms were taken from the algorithms found in Press, Teukolsky, Vetterling, and Flannery (1992).

Parameter estimates, $R M S D \mathrm{~s}$, and proportion of variance accounted for $\left(R^{2}\right)^{10}$ for each participant are provided in Table 4 (criterion-calibration model) and Table 5 (SOCR). Although comparing models with different numbers of parameters with a statistical control for model complexity is often recommended (e.g., Pitt \& Myung, 2002), this proved unnecessary, since the smaller model (criterion-calibration model) always had a better fit than the larger model (SOCR). ${ }^{11}$

For the criterion-calibration model (Table 4), the poorest fit is for participant A.B., and this fit still accounts for almost 95\% of A.B.'s actual data. For two participants, M.C. and S. A., the model accounts for over $99 \%$ of the actual variability. Fig. 5 shows the fit of the criterion-calibration model to the data reported by Siegel et al. (2009). Target $\Delta P$ is on the $x$ axis, and the probability of a "strong" response is on the $y$ axis. The markers indicate the actual data, and the lines indicate the simulated data. The triangles represent the target psychometric functions; the open triangles are from the companion $_{0}$ streams (target ${ }_{0}$ function), and the filled triangles are from the companion ${ }_{1}$ streams (target $_{1}$ function). The circles represent the companion psychometric functions; the open circles are from the companion streams (companion $_{0}$ function), and the filled circles are the companion $_{1}$ streams (companion $_{1}$ function).

Figure 5 shows the tightness of the fit between simulated and actual data, with the lines of the simulated data often

\footnotetext{
${ }^{10}$ The use of $R^{2}$ is problematic for proportional data, such as ours, for least-squares regression models. Ours, however, is not a regression model, but a mechanism, or process model, and it is not fit by a leastsquares method. Of course, $R^{2}$, calculated here as $1-\mathrm{SS}_{\mathrm{Error}} / \mathrm{SS}_{\mathrm{Total}}$, is influenced by the variance in the original data, shrinking as $\mathrm{SS}_{\text {Total }}$ shrinks; if $\mathrm{SS}_{\text {Total }}$ is small enough, $R^{2}$ can even become negative when $\mathrm{SS}_{\text {Error }}$ is high. Nonetheless, since the two models examined in this article are applied to the same data sets, the variance in the data is the same for both models, and the reduction in size of $R^{2}$ with data variance works against both models. The intuitive nature of $R^{2}$, as well as its broad familiarity, makes it useful as a means of assessing fit.

${ }^{11}$ Model complexity, as Pitt and Myung (2002) pointed out, is not simply a matter of the number of parameters, but also reflects the complexity of the functions. However, statistical controls such as the Akaike information criterion (e.g., Burnham \& Anderson, 2004) control only for number of parameters, and not for differences in function complexity.
}

running almost perfectly over the markers of the actual data. That the fit to the data is consistently good is notable, given the wide range of data patterns the model is called on to fit. A.S., for example, shows almost no cue interaction effect, with target cue functions (triangle markers) that nearly fall on top of one another, while X. G. and M.C. show target functions that are widely separated. Both A.S. and S.A. exhibit very flat companion functions (circle markers), while X.G.'s companion $_{0}$ function (open circles) has a pronounced negative slope, yet X.G.'s companion ${ }_{1}$ function (filled circles) is flat.

The model yields primarily positive values for the SAF parameters, although 2 participants (A.S. and C.H.) each produce a negatively valued SAF parameter. A negatively valued SAF would imply that participants were moving the criterion away from signals. For both participants, the negative values are close to zero ( -0.01 and -0.40 , respectively), and we suspect that they are not meaningfully different from zero, thus representing an unwillingness to move the criterion. We refit the model for both C.H. and A. S., adding in the constraint that neither SAF parameter could be less than zero. For both participants, the model still accounted for over $90 \%$ of the variance, with most parameters other than the originally negative SAF parameters changing slightly. For C.H., the refit parameters changed to $\mathrm{SAF}_{0}=0.00, \mathrm{SAF}_{1}=2.00$, and $\sigma=0.30$, and the $R M S D$ changed to 0.080 , with $R^{2}$ changing to .915 . For A. $\mathrm{S}$., the refit parameters changed to $\mathrm{SAF}_{0}=1.93, \mathrm{SAF}_{1}=$ 0.00 , and $\sigma=0.48$, and the $R M S D$ changed to 0.065 , with $R^{2}$ changing to .913 .

For the SOCR model (Table 5), X.G. yields the worst fit; the model accounts for just under $90 \%$ of X.G.'s data, as compared with $98 \%$ for the criterion-calibration model. This mismatch between data and simulation is clear in Fig. 6. SOCR does much better, however, for the other participants, accounting for $92 \%-99 \%$ of the remaining participants' data. Looking at Fig. 6, a pattern emerges of the model's producing cue interaction effects smaller than those yielded by either the criterion-calibration model or the actual data, and there are trends toward producing flat companion $_{0}$ functions and negatively sloped companion ${ }_{1}$ functions. This would explain why the model would have its greatest difficulty fitting X.G.'s data, which display the opposite trends.

Overall, the cue interaction effect in Siegel et al. (2009) data is well described in terms of people's shifting their report criterion after evaluating the contingency of each cue while maintaining a constant sensitivity. The criterioncalibration model describes this criterion shift as involving a nonlinear weighting of the distance between the subjective value of a stimulus and the report criterion, yielding substantial shifts only in response to stimuli far from 
Table 5 Estimated parameter values and fit indices for the SOCR model, all participants, Siegel et al. (2009)

\begin{tabular}{|c|c|c|c|c|c|c|c|}
\hline \multirow[b]{2}{*}{ Participant } & \multicolumn{5}{|c|}{ Parameters } & \multirow[b]{2}{*}{$R M S D$} & \multirow[b]{2}{*}{$R^{2}$} \\
\hline & $s 1$ & $s 2$ & $s 3$ & $k 1$ & $k 2$ & & \\
\hline X.G. & 1.000 & 1.000 & 0.145 & 0.381 & 0.749 & .125 & .897 \\
\hline M.C. & 1.000 & 1.000 & 0.170 & 0.583 & 0.723 & .076 & .964 \\
\hline A.S. & 0.691 & 0.541 & 0.429 & 0.116 & 0.039 & .051 & .950 \\
\hline A.B. & 0.541 & 0.571 & 0.369 & 0.193 & 0.145 & .077 & .918 \\
\hline S.A. & 0.529 & 0.426 & 0.422 & 0.372 & 0.389 & .069 & .961 \\
\hline C.H. & 0.575 & 0.145 & 0.388 & 0.263 & 0.318 & .033 & .985 \\
\hline
\end{tabular}

"s 1, " " $s 2$," and " $s 3$ " are saliency parameters for the companion, outcome, and target, respectively; " $k 1$ " is a decrement parameter that controls the reduction in associative strength between two events when only one of the two is present, and " $k 2$ " is a competition parameter controlling the extent to which the comparator term reduces responding. Actual and simulated data are shown in Fig. 6

criterion. The data from Siegel et al., and possibly from other cue interaction studies, appear to reflect an attempt to reduce error in the placement of a report criterion by making use of the relative "surprisingness" of contingencies - that is, how far from the criterion a contingency is.
That X.G.'s data are clearly more problematic for the SOCR model (Stout \& Miller, 2007) than for the criterioncalibration model suggests an avenue for exploring the differences between the two models. The SOCR model tends to produce negatively sloped companion $_{1}$ functions
Fig. 5 Simulated (criterioncalibration model) and actual data reported in Siegel et al. (2009). Markers indicate actual data; lines indicate simulated data

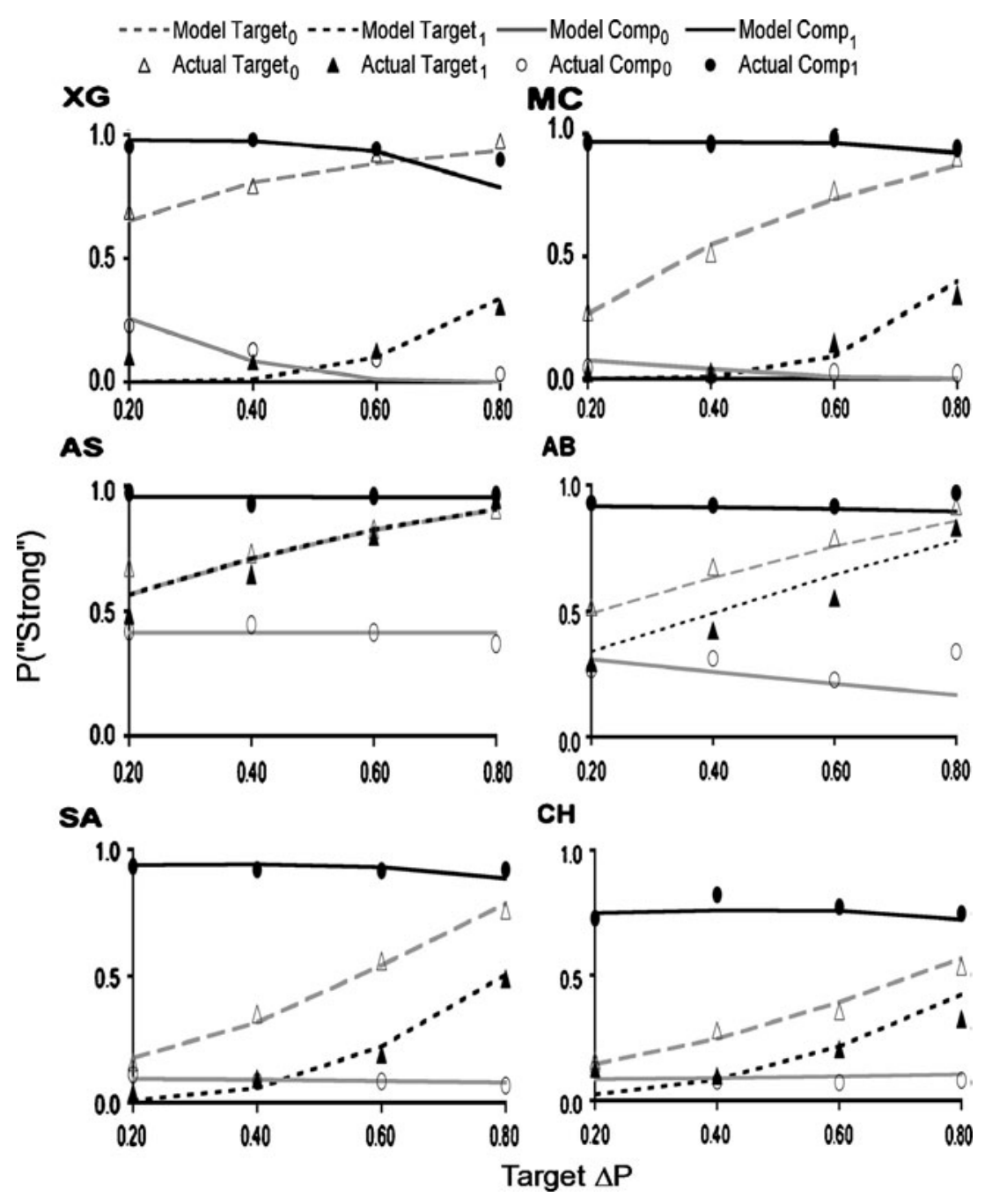


Fig. 6 Simulated (SOCR model) and actual data reported in Siegel et al. (2009). Markers indicate actual data; lines indicate simulated data

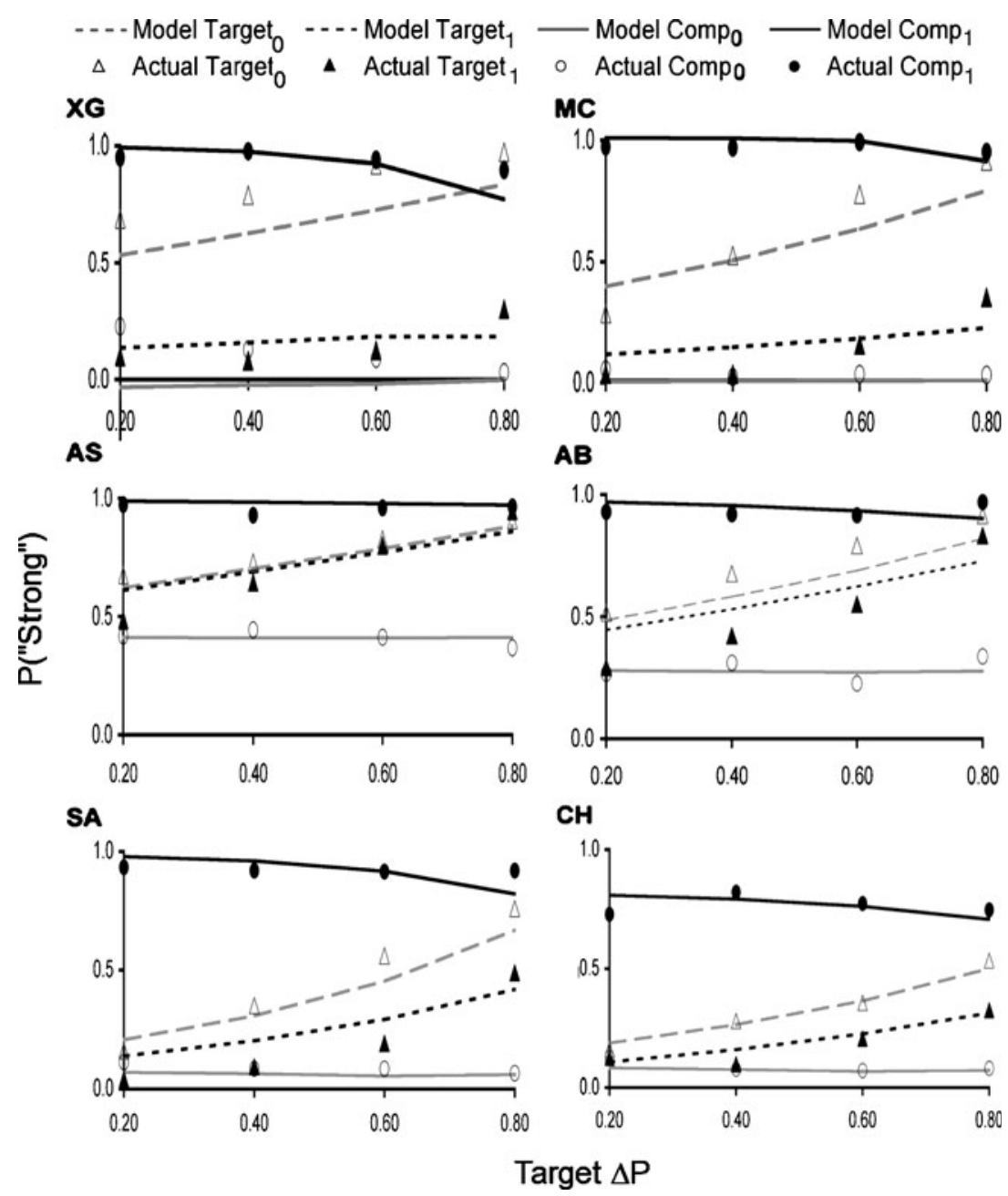

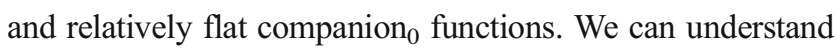
this pattern by thinking back to the basic principles of the model-namely, that responding is a function of the relative strength of associations. In the SOCR model, a measured cue's response strength is reduced by the strength of the comparator function - the rival cues' association with the outcome multiplied by the rival cues' association with the measured cue, given in Eq. 4 by the term within parentheses.

If we substitute $\Delta P$ for the associative strength measures SOCR actually computes - in Siegel et al. (2009) data the two are correlated-we see that the cue-cue contingencies, $P$ (Target $\mid$ Companion $)-P$ (Target $\mid \sim$ Companion $)$, differ considerably between the companion ${ }_{0}$ and companion streams,

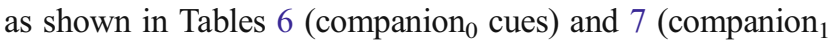

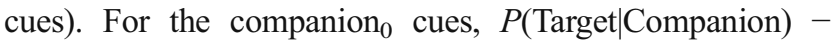
$P($ Target $\mid \sim$ Companion) is largely constant at $\Delta P(.4)$, dropping slightly to $\Delta P(.2)$ when the target cue has a contingency with the outcome of $\Delta P(.8)$. For the companion $_{1}$ cues, however, $P$ (Target $\mid$ Companion $)-P($ Target $\mid \sim$ Companion $)$ matches that of the contingency between target and outcome, because the companion is perfectly correlated with the outcome. Thus, for companion $_{0}$ cues, the comparator function is related to the companion $\Delta P$ value multiplied by a nearly constant value (the cue-cue contingency), whereas for companion ${ }_{1}$ cues, the comparator function is related to the companion $\Delta P$ value multiplied by the target $\Delta P$ value and, so, the value of the comparator function increases as target $\Delta P$ increases. The companion ${ }_{1}$ cue elicits a markedly greater decrease in responding as target $\Delta P$ increases.

SOCR (Stout \& Miller, 2007) predicts that reciprocal cue interaction effects-with the target $\Delta P$ influencing com-

Table 6 Companion-target contingencies and related cell frequencies for companion ${ }_{0}$ cues in Siegel et al. (2009)

\begin{tabular}{|c|c|c|c|c|c|c|c|c|}
\hline & \multicolumn{8}{|c|}{ Target Cue Contingency } \\
\hline & \multicolumn{2}{|l|}{.2} & \multicolumn{2}{|l|}{.4} & \multicolumn{2}{|l|}{.6} & \multicolumn{2}{|l|}{.8} \\
\hline & $B$ & $\sim B$ & $B$ & $\sim B$ & $B$ & $\sim B$ & $B$ & $\sim B$ \\
\hline$A$ & 14 & 6 & 14 & 6 & 14 & 6 & 12 & 8 \\
\hline$\sim A$ & 6 & 14 & 6 & 14 & 6 & 14 & 8 & 12 \\
\hline$\Delta P$ & .4 & & .4 & & .4 & & .2 & \\
\hline
\end{tabular}

$A$ denotes the companion cue, and $B$ denotes the target cue 
Table 7 Companion-target contingencies and related cell frequencies for companion ${ }_{1}$ cues in Siegel et al. (2009)

\begin{tabular}{|c|c|c|c|c|c|c|c|c|}
\hline & \multicolumn{8}{|c|}{ Target Cue Contingency } \\
\hline & \multicolumn{2}{|l|}{.2} & \multicolumn{2}{|l|}{.4} & \multicolumn{2}{|l|}{6} & \multicolumn{2}{|l|}{.8} \\
\hline & $B$ & $\sim B$ & $B$ & $\sim B$ & $B$ & $\sim B$ & $B$ & $\sim B$ \\
\hline$A$ & 12 & 8 & 14 & 6 & 16 & 4 & 18 & 2 \\
\hline$\sim A$ & 8 & 12 & 6 & 14 & 4 & 16 & 2 & 18 \\
\hline$\Delta P$ & .2 & & .4 & & .6 & & .8 & \\
\hline
\end{tabular}

$A$ denotes the companion cue, and $B$ denotes the target cue

panion ratings, as well as the reverse - are always present, if negligibly so. It also predicts an asymmetry in these

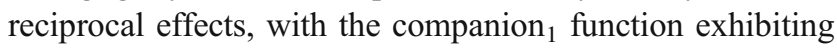
a much larger effect than the companon ${ }_{0}$ function. In contrast, the criterion-calibration model predicts that such reciprocal cue interaction effects are manifest only when the criterion is pulled over the subjective range of companion contingencies. This can happen either with very large values of SAF, relative to the variance of processing, or when the range of companion cue contingencies is made to overlap that of the target cue contingencies. We explored these ideas in Experiment 1.

\section{Experiment 1: Close companions}

In Siegel et al. (2009), the cue interaction effect was mainly one-way; the companion contingency affected responding to the target contingency, but the target contingency had little effect on responding to the companion contingency. The criterion-calibration model predicts that one-way cue interaction effects emerge given the extreme companion contingencies used by Siegel et al. Because the criterion is pulled toward the center of the experienced range of contingencies, extreme values will usually fall outside the range over which the criterion roams. The subjective companion contingency values from companion streams (i.e., $\Delta P=0$ ) will thus almost always fall below the criterion, yielding flat companion functions. Similarly, subjective companion contingencies from companion $_{1}$ streams (i.e., $\Delta P=1$ ) will almost always fall above the criterion, again yielding flat companion functions.

According to the criterion-calibration model, a reciprocal cue interaction effect should appear as the degree of overlap between companion and target contingency ranges increases. In Experiment 1, the $\Delta P$ values of the companion cues were set to $\Delta P(.2)$ and $\Delta P(.8)$. Not only should reciprocal cue interactions emerge, but also they should be approximately symmetrical effects, with both companions being equally affected by changes in target $\Delta P$.
In contrast, according to SOCR, by increasing the value of the weaker companion contingency, we are making it more robust to the effects of its rival cue, the target cue, but the comparator function increases slightly due to a stronger companion-target contingency. We are weakening the stronger companion's value, while its contingency with the target cue - and thus its comparator function - still increases with target $\Delta P$, just as in Siegel et al. (2009) experiment. Responding thus would be expected to decrease just as sharply with target $\Delta P$ for the strong companion cue. Thus, overall levels of responding for companion $n_{0}$ and companion ${ }_{1}$ functions should shift up and down, respectively, when companion contingencies are changed from $\Delta P(0.0)$ and $\Delta P(1.0)$ to $\Delta P(.2)$ and $\Delta P(.8)$, but the asymmetry that SOCR predicted for the Siegel et al. data should remain.

\section{Method}

\section{Participants}

We recruited 4 participants from within the Department of Psychology, Neuroscience, and Behaviour, at McMaster University; 3 were graduate students, and the 4 th was a postdoctoral researcher. All the participants were paid \$10/ session. Two of these participants, A.B. and X.G., also participated in the experiment reported by Siegel et al. (2009).

Apparatus and materials

We used stimuli identical to those used in Siegel et al. (2009) (Fig. 1b). Cues were a blue square and a blue triangle, and the outcome was a red circle. Stimulus displays were created by the factorial combination of three pairs of binary events: red circle/no circle, blue square/no square, and blue triangle/no triangle. The resulting eight possible cue-outcome stimulus triplets are shown in Fig. 7. The location of each shape, when it was present, was constant across all frames: The red circle was centered above the blue shapes, the blue square was on the left, and the blue triangle was on the right. The blue square was always the target cue, and the blue triangle was always the companion. ${ }^{12}$ The geometric forms were presented on a gray background $(8.8 \mathrm{~cm}$ in height and $7.0 \mathrm{~cm}$ in width). The blue square measured $2.1 \mathrm{~cm}$ in height and width; the blue triangle measured $2.7 \mathrm{~cm}$ at its base and extended $2.3 \mathrm{~cm}$ in height; the red circle measured $2.5 \mathrm{~cm}$ in diameter. MetaCard software controlled stimulus presentation and data collection.

Streams were composed so that the target cue had a contingency with the circle of $\Delta P(.2), \Delta P(.4), \Delta P(.6)$, or $\Delta P$

\footnotetext{
${ }^{12}$ In Hannah et al. (2009), we counterbalanced shape across cue type (target, companion) and found little effect of shape. In most experiments, no effect was found, and the few obtained effects did not show a consistent pattern, nor did they influence the interpretation of other effects.
} 


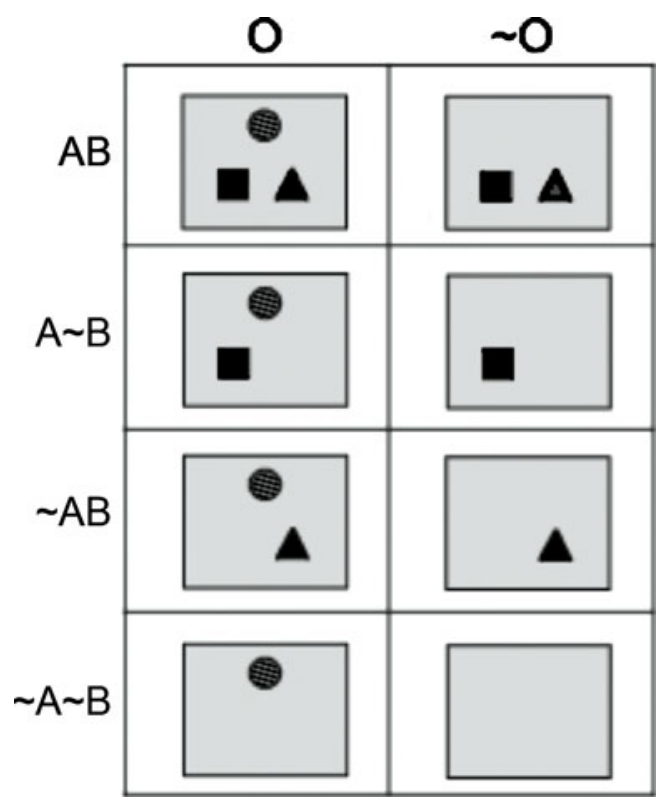

Fig. 7 The eight cue-outcome stimulus displays composing each streamed trial in Siegel et al. (2009) and Experiment 1

(.8), while the companion cue had a contingency with the circle of either $\Delta P(.2)$ or $\Delta P(.8)$. The $2 \times 4$ combination of companion and target values created eight types of streamed trials, each defined by one of the $4 \times 2$ contingency matrices in Tables 8 (companion $_{.2}$ streams) and 9 (companion $_{.8}$ streams). Each streamed trial consisted of 40 presentations, or frames, of the eight cue-outcome combinations summarized in these matrices.

\section{Procedure}

The experimenters explained to participants that they were to judge whether the contingency between one of two blue shapes and a red circle presented in a streamed trial was weak or strong. Participants ran in 15 sessions consisting of five blocks, with each block consisting of 48 streamed trials. Participants saw the eight types of streamed trials in a randomized order within blocks.

Table 8 Four contingency matrices defining the different stream types for companion 0.2 streams used in Experiment 1

\begin{tabular}{|c|c|c|c|c|c|c|c|c|}
\hline & \multicolumn{8}{|c|}{ Target Cue Contingency } \\
\hline & \multicolumn{2}{|l|}{0.2} & \multicolumn{2}{|l|}{0.4} & \multicolumn{2}{|l|}{0.6} & \multicolumn{2}{|l|}{0.8} \\
\hline & $O$ & $\sim O$ & $O$ & $\sim O$ & $O$ & $\sim O$ & $O$ & $\sim O$ \\
\hline$A B$ & 10 & 6 & 11 & 5 & 12 & 4 & 12 & 2 \\
\hline$A \sim B$ & 2 & 2 & 1 & 3 & 0 & 4 & 0 & 6 \\
\hline$\sim A B$ & 2 & 2 & 3 & 1 & 4 & 0 & 6 & 0 \\
\hline$\sim A \sim B$ & 6 & 10 & 5 & 11 & 4 & 12 & 2 & 12 \\
\hline
\end{tabular}

$A$ denotes the companion cue, and $B$ denotes the target cue
Table 9 Four contingency matrices defining the different stream types for companion ${ }_{0.8}$ streams used in Experiment 1

\begin{tabular}{|c|c|c|c|c|c|c|c|c|}
\hline & \multicolumn{8}{|c|}{ Target Cue Contingency } \\
\hline & \multicolumn{2}{|l|}{0.2} & \multicolumn{2}{|l|}{0.4} & \multicolumn{2}{|l|}{0.6} & \multicolumn{2}{|c|}{0.8} \\
\hline & $O$ & $\sim O$ & $O$ & $\sim O$ & $O$ & $\sim O$ & $O$ & $\sim O$ \\
\hline$A B$ & 10 & 0 & 12 & 0 & 14 & 0 & 16 & 0 \\
\hline$A \sim B$ & 8 & 2 & 6 & 2 & 4 & 2 & 2 & 2 \\
\hline$\sim A B$ & 2 & 8 & 2 & 6 & 2 & 4 & 2 & 2 \\
\hline$\sim A \sim B$ & 0 & 10 & 0 & 12 & 0 & 14 & 0 & 16 \\
\hline
\end{tabular}

$A$ denotes the companion cue, and $B$ denotes the target cue

Each frame in a stream was presented for $100 \mathrm{~ms}$, with a 100-ms interframe interval, for a stream duration of $7.9 \mathrm{~s}$. Participants judged the contingency either between the square and circle or between the triangle and circle, reporting their decision by clicking one of the two buttons (labeled "Weak" or "Strong") on the computer monitor. We directed participants which cue-outcome contingency to report by showing a small picture of the focal pair at the end of each stream. Participants reported on the target contingency for half of trials in a session and on the companion contingency for the other half.

\section{Results and discussion}

As Fig. 8 shows, the performance of participants in Experiment 1 (open and filled markers) shows the same general pattern of results as that found in Siegel et al. (2009). Target functions (triangle markers) show widely divergent values at the PSE- the cue interaction effect. As Table 10 shows, these results are again well fit by the criterion-calibration model (with no negative SAF values), which accounted for at least $94 \%$ of actual variance for all the participants but X.G., for whom nearly $90 \%$ of actual variance was accounted for. That the model accounts for less than $90 \%$ of X.G.'s data is comforting, given the very close fits in the previous data set, since this shows that the model is not overfitting the data and accounting for all data patterns, including impossible ones. Overall, our participants, like those in Siegel et al., produced a cue interaction effect that is expressed as a change in report criterion, rather than sensitivity, and that is well accounted for by the criterion-calibration model.

In contrast, the fit of the SOCR model to the new data, shown visually in Fig. 9, is worse overall than its fit to Siegel et al. (2009) data. As an examination of Table 11 shows, no participants in Experiment 1 had even $90 \%$ of their data accounted for by the SOCR model. Another effect of making the contingencies of companions and targets overlap concerns the relative saliency of the cues as 
Fig. 8 Simulated (criterioncalibration model) and actual data for all participants, Experiment 1. Markers indicate actual data; lines indicate simulated data
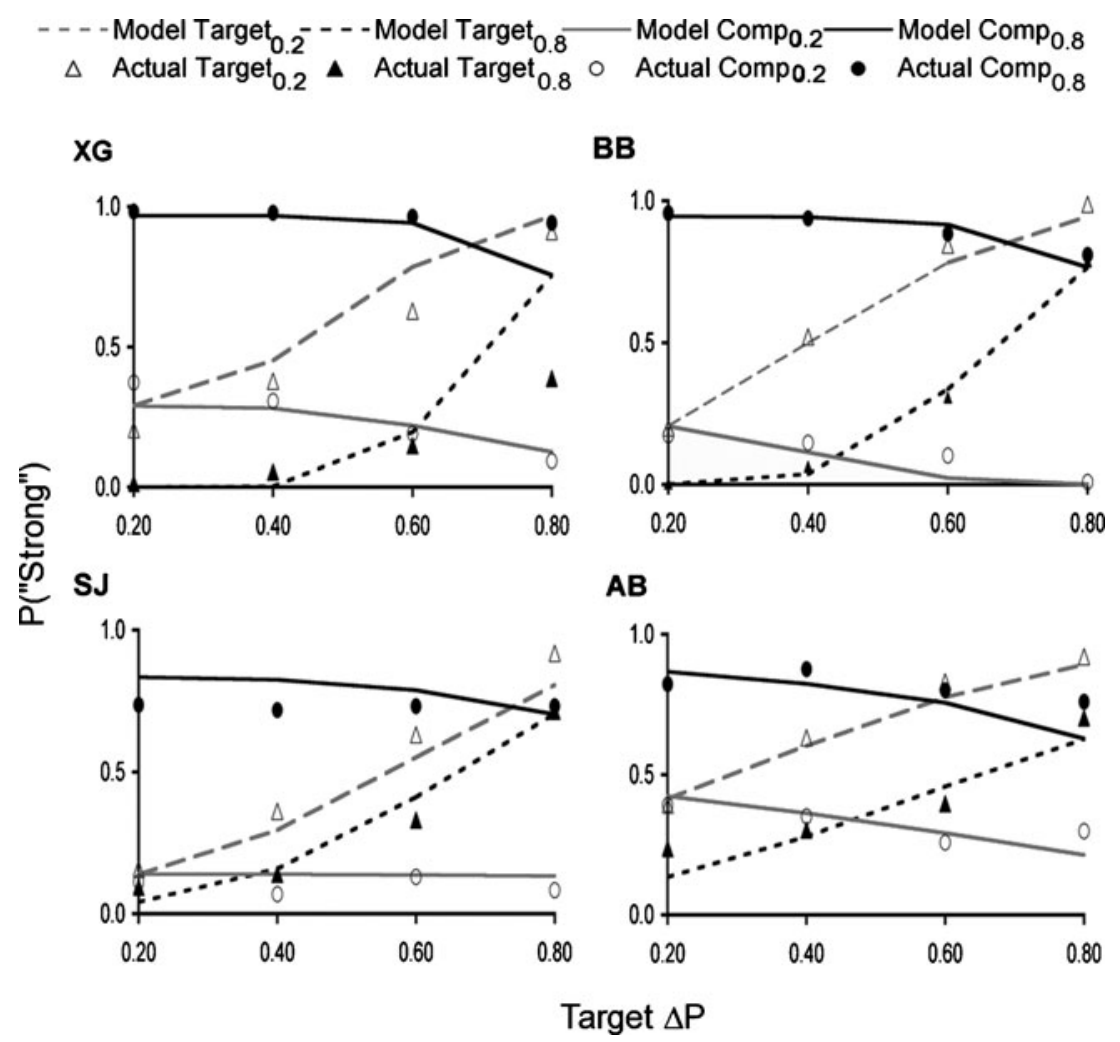

estimated by SOCR. In the previous simulation, companion cues were always more salient than target cues $(s 1>s 3$ for all participants); after the contingencies were made to overlap in Experiment 1, the enhanced saliency for the companion cues disappeared. It is possible that the enhanced saliency for the companion cues in the first study reflected the greater discriminability of perfectly contingent cues over other cues.

The purpose of Experiment 1, however, was to test the prediction that when the companion contingencies were made to overlap with the extremes of the target contingency range, companion cues would begin to manifest a cue interaction effect in the form of negatively sloped companion functions. Figs. 8 and 9 show noticeably negative-going

Table 10 Estimated parameter values for criterion-calibration model and fit indices, Experiment 1

\begin{tabular}{|c|c|c|c|c|c|}
\hline \multirow[b]{2}{*}{ Participant } & \multicolumn{3}{|c|}{ Parameters } & \multirow[b]{2}{*}{$R M S D$} & \multirow[b]{2}{*}{$R^{2}$} \\
\hline & $\mathrm{SAF}_{0}$ & $\mathrm{SAF}_{1}$ & $\sigma$ & & \\
\hline X.G. & 1.21 & 5.15 & 0.06 & 0.119 & .888 \\
\hline B.B. & 3.39 & 3.96 & 0.10 & 0.035 & .991 \\
\hline S.J. & 0.11 & 2.95 & 0.20 & 0.064 & .954 \\
\hline A.B. & 1.63 & 4.39 & 0.25 & 0.061 & .938 \\
\hline
\end{tabular}

"SAF" indicates the SAF parameter for companion streams. "SAF $_{1}$ " indicates the SAF parameter for companion ${ }_{1}$ streams. Actual and simulated data are shown in Fig. 8 companion slopes (circle markers) for all the participants in Experiment 1 but one (S.J.), while flat slopes predominate in Figs. 5 and 6 (Siegel et al. 2009, data).

The best-fitting regression slopes for companion functions in both experiments are summarized in Table 12. The slopes of the companion functions found in Experiment 1 are more negative than those found in Siegel et al. (2009) data. Furthermore, the slopes of the Experiment 1 companions are uniformly negative, whereas in Siegel et al.'s data, some slopes are actually positive, albeit negligibly so, suggesting that the Siegel et al.'s companion slopes are showing only random fluctuations around zero. An exact permutation test (Mewhort, Kelly \& Johns, 2009) was conducted to compare the companion slopes for the new data with those for Siegel et al.'s. For both the small (0.0/.2) and the large (1.0/.8) companion functions, the slopes produced in Experiment 1 were reliably more negative than those in Siegel et al.'s experiment, $p=.024$ and .019 , respectively.

Reciprocal cue interaction effects of equivalent magnitude emerged for both companion ${ }_{0.2}$ and companion $_{0.8}$ functions. It would seem that reciprocal cue interactions in a psychophysical paradigm are symmetric and require some overlap between companion and target contingency ranges, as predicted by the criterion-calibration model. Neither the symmetry nor the overlap dependency is consistent with SOCR (Stout \& Miller, 2007). As is clear in Fig. 9, SOCR predicts flat functions for the companion $_{0.2}$ function. The absence of this asymmetry in the actual data likely accounts 
Fig. 9 Simulated (SOCR model) and actual data for all participants, Experiment 1. Markers indicate actual data; lines indicate simulated data

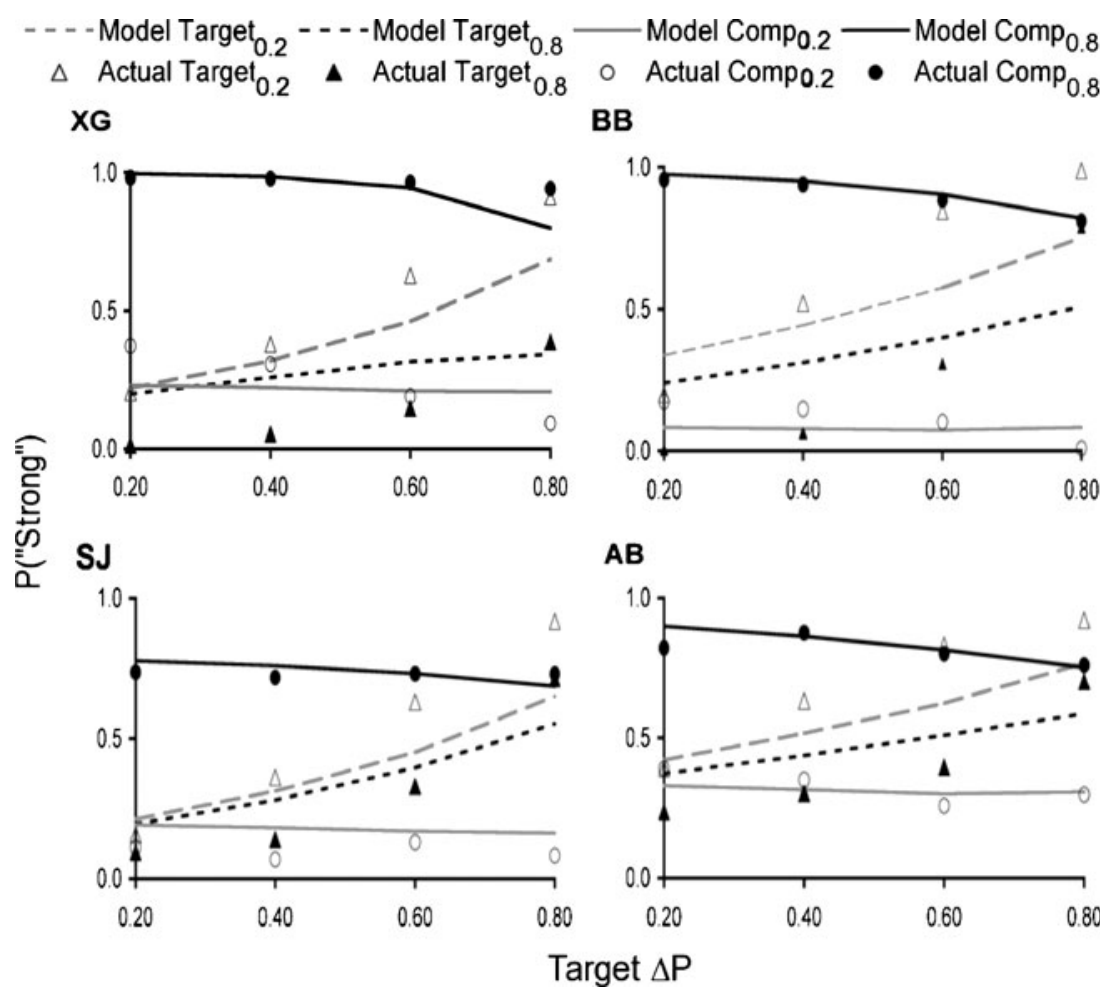

for the SOCR model's poorer fit to the present data, as compared with that for the Siegel et al. (2009) data set.

\section{General discussion}

The criterion-calibration model of cue interaction in contingency judgment captured most of the variability in found in Siegel et al.'s (2009) data and in Experiment 1. At worst, the model accounted for just under $89 \%$ of a participant's data (X.G., Experiment 1). The model accounted for over $93 \%$ for all other data sets and almost perfectly simulated 3 participants' performance (S.A. and M.C. in Siegel et al., 2009, B.B. in Experiment 1). The cue interaction effect, as manifested in psychometric functions, is correctly described in the criterion-calibration model as a shift in criterion (PSE), rather than as a difference in slope. With the exception of Miller and colleague's comparator account (Miller \& Matzel, 1988; Stout \& Miller, 2007), other accounts describe cue interaction effects as differences in signal acquisition and, thus, predict differences in the slope of psychometric functions for targets with companions of different contingencies.

The model also correctly predicted the novel finding that when companion-cue contingencies overlap those of target-cue contingencies, companion functions will exhibit a symmetric cue interaction effect. For all but 1 participant in Experiment 1, the probability of calling the companion's contingency "Strong" decreased as the target cue's contingency increased. As predicted by the model, the decline in companion responding was similar for both companion functions.

Table 11 Estimated parameter values for SOCR model and fit indices, Experiment 1

\begin{tabular}{lllllll}
\hline & \multicolumn{2}{l}{ Parameters } & & & & \\
\cline { 2 - 5 } Participant & $s 1$ & $s 2$ & $s 3$ & $k 1$ & $k 2$ & $R M S D$ \\
\hline X.G. & 0.414 & 1.000 & 0.516 & 0.320 & 0.752 & .125 \\
B.B. & 0.624 & 0.536 & 0.380 & 0.288 & 0.344 & .153 \\
S.J. & 0.307 & 0.250 & 0.493 & 0.283 & 0.211 & .112 \\
A.B. & 0.295 & 0.565 & 0.315 & 0.218 & 0.364 & .099 \\
\hline
\end{tabular}

"s1," "s2," and "s3" are saliency parameters for the companion, outcome, and target, respectively; " $k 1$ " is a decrement parameter controlling the reduction in associative strength between two events when only one of the two is present, and " $k 2$ " is a competition parameter controlling the extent to which the comparator term reduces responding. Actual and simulated data are shown in Fig. 9 
Table 12 Best-fitting regression slopes for companion functions, Siegel et al. (2009) and Experiment 1

\begin{tabular}{llll}
\hline Experiment & Participant & Companion0/.2 & Companion1/.8 \\
\hline Siegel et al. & X.G. & -0.060 & -0.020 \\
& M.C. & -0.005 & -0.004 \\
& A.S. & -0.02 & 0.0004 \\
& A.B. & 0.013 & 0.012 \\
& S.A. & -0.013 & -0.004 \\
Experiment 1 & C.H. & -0.014 & 0.001 \\
& X.G. & -0.478 & -0.067 \\
& B.B. & -0.268 & -0.247 \\
& S.J. & -0.012 & -0.001 \\
& A.B. & -0.186 & -0.131 \\
\hline
\end{tabular}

The criterion-calibration model also captures the flatness of the companion cue functions when the value of their objective contingency falls outside the range of the target contingencies, as in Siegel et al. (2009). It should be noted that the flatness of companion slopes in Siegel et al.'s data could not be fit with conventional signal detection models, since the slope reflects the reciprocal of the variance of the distributions and a flat slope would require constant responding - that is, zero variance. Of course, not only is zero variance implausible, zero variance would yield an infinite slope, given that slope is the reciprocal of variance.

Perhaps most important, the criterion-calibration model allows us to describe the process by which cue interaction effects emerge. Many signal detection models are measurement models, confined to describing the relations among processes, leaving an account of mechanisms unaddressed. The criterion-calibration model, therefore, accounts for all aspects of the psychometric functions produced in a cue interaction task and extends signal detection theory to explaining the processing underlying these functions. It yields testable predictions, as in Experiment 1, allowing us to examine questions such as how contingency judgments would change with manipulations that change sensitivity or the plausibility of distant contingencies as evidence of criterion shift or when parameters are held constant and inputs are manipulated.

The use of a binary response is required to obtain a conventional psychometric function, precluding the rating scales typically used in contingency and causality judgment research (see Allan et al., 2008, for a detailed justification for the use of binary response in contingency research). Extending the model to fit the more conventional rating scales is possible in principle, however, given some assumptions relating the distance between a contingency event and the criterion to distance along the response scale.
This requires not only some additional assumptions to describe the mapping of the contingency criterion distance to a rating distance, but also some principled way of relating criterion-and thus, distance from criterion-to some anchor point on the scale.

Criterion calibration and the comparator hypothesis

The only other model readily capable of accounting for cue interactions as a post-acquisition phenomenon, Stout and Miller's (2007) SOCR, does a good job of accounting for Siegel et al.'s (2009) data but yields poorer fits than the criterion-calibration model for all participants. For the data in Experiment 1, the SOCR model is unable to account for as much as $90 \%$ for any participant. Furthermore, the SOCR model incorrectly predicts that asymmetric reciprocal cue interaction effects should be present regardless of the degree of overlap between target and companion contingencies.

Cue interaction effects emerge in both models as a postacquisition change elicited by a comparison process. The comparator model does account for over $90 \%$ of most of Siegel et al.'s (2009) data and for over $80 \%$ of all the data. This is an impressive level of performance for a model applied to an experimental situation far removed from the animal learning situations it was designed to explain. That both models provide reasonable fits to the data and have effects emerge out of a postacquisition comparison process provides converging support for the postacquisition interpretation of cue interaction effects.

It is interesting to speculate on the relations that the two models may share. One way of viewing the comparator hypothesis is that it calls for comparing a signal against a criterion, where the criterion is the comparator term - that is, the rival associations. If this is a valid analysis, we can see the comparator hypothesis/SOCR model as a kind of signal detection model. The criterion would change as the measured cue changed, since changing which cue was measured would change what terms go into the comparator, and as the strength of rival cues changed. Therefore, the SOCR model could be conceptualized as a dynamic signal detection model, like the criterion-calibration model, but with a different set of dynamics controlling criterion shifts. Such a framing would entail a criterion that is generated by the same processes as those that generate the signal. Whether this violates a key assumption of signal detection theory - that criterion and sensitivity are independent - is unclear. That criterion and sensitivity are generated by the same processes suggests that it does violate this tenet. The noncompetitive nature of association formation within the SOCR model, however, allows for the value of a signal to change without any change to rival terms making up the criterion, suggesting that criterion and sensitivity in this 
framing are statistically independent, even if causally related. Regardless of these differences, there remains the intriguing possibility that both models are special cases of some more general set of mechanisms.

As compared with the SOCR model, the cue calibration model, in its current formulation, is of limited generality. SOCR has been shown to simulate a wide range of conditioning phenomena. The inclusion of saliency parameters, for example, allows SOCR to describe saliency effects linked to sensory modality, important to a large class of overshadowing effects studied in the animal learning literature. The criterion-calibration model would be unable to account for such effects without additional parameters, thus diminishing any advantage over SOCR in terms of having a small parameter set.

Integrating associative and reasoning perspectives

In Allan et al. (2008), and in Siegel et al. (2009), the authors argued that signal detection theory and associative learning could be integrated into a single account that describes associative processes as the means by which a contingency or causality signal is extracted. Although the role that higher order, inferential processes could play was not discussed, it is tempting to allow such processes to influence the positioning of a report criterion, influenced as it is by expectations, strategies, and other higher order constructs.

Knowledge and expectations are known to influence contingency-related decisions such as causal judgments in a very flexible manner (e.g., Buehner \& May, 2004; Mitchell, Killedar \& Lovibond, 2005), and such flexibility becomes even easier to integrate into a signal detection framework if the criterion is understood to be intrinsically dynamic. In the criterion-calibration model, any knowledge, expectation, and so forth that influence the plausibility that distance from that criterion reflects an error in criterion placement will have a large effect on responding. For example, although we have assumed that people try to maintain an unbiased (mid-range) criterion, expectations or beliefs could shift the preferred criterion location that people try to maintain from the mid-range position we have assumed. Maintaining a position away from the middle of the range may require asymmetric SAF terms, producing a drift toward a point away from the middle of the range. Alternatively, how the situation is construed could influence the willingness to ascribe a deviation from criterion as an error in criterion; that is, the size of the SAF term may be influenced by how the situation is interpreted. For example, if people in a causality judgment task are confronted with a scenario that violates causal expectations, the resulting contingency signal may be discounted as an anomaly, resulting in no shift in criterion. Thus, asymmetric
SAF terms may emerge for belief-consistent and beliefinconsistent situations.

Comparison with other criterion-shifting models

Ours is not the first model describing decisions emerging as a result of a variable report criterion. Parducci's (1965) range-frequency theory explored this general notion. Parducci described decisions emerging from a report criterion (an "empirical limen") that emerged by integration of two other criteria, one influenced by the range of experienced values and the other by the frequency of experienced values. Each of these criteria changes with differences in experienced range and frequency, pulling the "empirical limen," or observed report criterion, in different directions. Not only do we employ a single criterion, but the dynamics of its shifting are clearly different from those of Parducci's model.

The ideas formalized by Treisman (Treisman, 1984a, 1984b; Treisman \& Williams, 1984) bring us closer to the ideas embodied in the criterion-calibration model. However, although the two approaches share the assumption that criterion shifting represents an attempt by people to establish the optimum criterion for a context, there are a number of differences between our model and those of Treisman and colleagues. Treisman and Williams laid out three models, all of which made use of linear shift functions, ${ }^{13}$ and assumed that participants have a global criterion position that functions as an attractor, pulling a shifted criterion back toward it. In accounting for more complicated decisions, such as magnitude judgments, Treisman (1984b) required multiple such criteria, each marking off the boundary between two stimulus representations. In contrast, we have a single criterion shifted by a nonlinear operator, with no preferred position. Furthermore, the criterion moves away from stimuli in Treisman and colleagues' models, rather than toward stimuli, as in our model.

Our approach of treating the size of difference between a stimulus event and a criterion as an error signal has precedent in some of Thomas's (1975) models of probability matching. Thomas found that the best-fitting model for probability-matching situations where participants have no feedback was one in which a criterion moved toward a signal by a bounded, linearly scaled difference of the signal and criterion. The similarities with Thomas's model suggest that our model may embody principles that apply more broadly than just to cue interaction effects in a contingency

\footnotetext{
${ }^{13}$ Treisman (1984b) presented a more general form of his indicator trace model with a shift function having a variable exponent, allowing for a nonlinear shift function. However, such a nonlinear function was never implemented in Treisman's published simulations.
} 
judgment task. It is possible that our approach can apply to any circumstance in which people are making a decision across an unfolding range of values while trying to maintain a constant criterion position.

Memory and criterion shifts

In recent years, researchers in the field of memory have invoked criterion variability to explain memory phenomena, particularly in recognition tasks (e.g., Hicks \& Marsh, 1998; Hockley \& Niewiadomski, 2007; Singer, Gagnon \& Richards, 2002; Stretch \& Wixted, 1998; Verde \& Rotello, 2007). These memory theories invoke the notion of criterion shifts to explain phenomena; this interest in criterion shifts has led to reformulations of traditional signal detection theory to accommodate random criterion variability (e.g., Benjamin, Diaz \& Wee, 2009) and to models describing the dynamics of responding following criterion shifts (e.g., Brown \& Steyvers, 2005; Brown, Steyvers \& Hemmer, 2007). However, it has not led to models formalizing the mechanisms of systematic criterion shifts. Our model may prove useful for guiding such formal developments.

\section{References}

Allan, L. G., Hannah, S. D., Siegel, S., \& Crump, M. J. C. (2008). The psychophysics of contingency assessment. Journal of Experimental Psychology: General, 137, 226-243.

Baker, A. G., Mercier, P., Valle-Tourangeau, F., Frank, R., \& Pan, M. (1993). Selective associations and causality judgments: Presence of a strong causal factor may reduce judgments of a weaker one. Journal of Experimental Psychology: Learning, Memory, and Cognition, 19, 414-432.

Benjamin, A. S., Diaz, M., \& Wee, S. (2009). Signal detection with criterion noise: Applications to recognition memory. Psychological Review, 116, 84-115.

Blaisdell, A. P., Bristol, A. S., Gunther, L. M., \& Miller, R. R. (1998). Overshadowing and latent inhibition counteract each other: Support for the comparator hypothesis. Journal of Experimental Psychology: Animal Behavior Processes, 24, 335-351.

Brown, S., \& Steyvers, M. (2005). The dynamics of experimentally induced criterion shifts. Journal of Experimental Psychology: Learning, Memory, and Cognition, 31, 587-599.

Brown, S., Steyvers, M., \& Hemmer, P. (2007). Modelling experimentally induced strategy shifts. Psychological Science, 31, 587599.

Buehner, M. J., \& May, J. (2004). Abolishing the effect of reinforcement delay on human causal learning. Quarterly Journal of Experimental Psychology, 57B, 179-191.

Burnham, K. P., \& Anderson, D. R. (2004). Multimodel inference: Understanding AIC and BIC in model selection. Sociological Methods Research, 33, 261-304.

Crump, M. J. C., Hannah, S. D., Allan, L. G., \& Hord, L. K. (2007). Contingency judgements on the fly. Quarterly Journal of Experimental Psychology, 60, 753-781.

Denniston, J. C., Savastano, H. I., Blaisdell, A. P., \& Miller, R. R. (2003). Cue competition as a retrieval deficit. Learning \& Motivation, 34, 1-31.
Gallistel, C. R., \& Gibbon, J. (2000). Time, rate, and conditioning. Psychological Review, 107, 289-344.

Green, D. M., \& Swets, J. A. (1966). Signal detection theory and psychophysics. New York: Wiley.

Hannah, S. D., Crump, M. J. C., Allan, L. G., \& Siegel, S. (2009). Cue interaction effects in contingency judgments using the streamed-trials procedure. Canadian Journal of Experimental Psychology, 63, 103-112.

Hicks, J. L., \& Marsh, R. L. (1998). A decrement-to-familiarity interpretation of the revelation effect from forced-choice tests of recognition memory. Journal of Experimental Psychology: Learning, Memory, and Cognition, 24, 1105-1120.

Hockley, W. E., \& Niewiadomski, M. W. (2007). Strength-based mirror effects in item and associative recognition: Evidence for within-list criterion changes. Memory \& Cognition, 35, 679-688.

Killeen, P. R., Fetterman, J. G., \& Bizo, L. A. (1997). Time's causes. In C. M. Bradshaw \& E. Szabadi (Eds.), Time and behaviour: Psychological and neurobehavioural analyses (pp. 79-131). Amsterdam: Elsevier.

Laux, J. P., Goedert, K. M., \& Markman, A. B. (2010). Causal discounting in the presence of a stronger cue is due to bias. Psychonomic Bulletin \& Review, 17, 213-218.

Mewhort, D. J. K., Kelly, M., \& Johns, B. T. (2009). Randomization and the unequal-N/unequal-variance problem. Behavior Research Methods, 41, 664-667.

Miller, R. R., \& Matzel, L. D. (1988). The comparator hypothesis: A response rule for the expression of associations. In G. H. Bower (Ed.), The psychology of learning and motivation (Advances in research and theory, Vol. 22, pp. 51-92). San Diego: Academic Press.

Mitchell, C. J., Killedar, A., \& Lovibond, P. F. (2005). Inference-based retrospective revaluation in human causal judgments requires knowledge of within-compound relationships. Journal of Experimental Psychology: General, 31, 418-424.

Nelder, J. A., \& Mead, R. (1965). A simplex method for function minimization. Computer Journal, 8, 308-313.

Parducci, A. (1965). Category judgment: A range-frequency model. Psychological Review, 72, 407-418.

Pearce, J. M. (1994). Similarity and discrimination: A selective review and a connectionist model. Psychological Review, 101, 587-607.

Pineño, O. (2007). A response rule for positive and negative stimulus interaction in associative learning and performance. Psychonomic Bulletin \& Review, 14, 1115-1124.

Pitt, M. A., \& Myung, J. (2002). When a good fit can be a bad fit. Trends in Cognitive Sciences, 6, 441-445.

Press, W. H., Teukolsky, S. A., Vetterling, W. T., \& Flannery, B. P. (1992). Numerical recipes in FORTRAN; The art of scientific computing (2nd ed.). New York: Cambridge University Press.

Rescorla, R. A., \& Wagner, A. R. (1972). A theory of Pavlovian conditioning: Variations in the effectiveness of reinforcement and non-reinforcement. In A. H. Black \& W. F. Prokosy (Eds.), Classical conditioning II: Current research and theory (pp. 64-99). New York: Appleton-Century-Crofts.

Shanks, D. R. (2007). Associationism and cognition: Human contingency learning at 25. Quarterly Journal of Experimental Psychology, 60, 291-309.

Siegel, S., Allan, L. G., Hannah, S. D., \& Crump, M. J. (2009). Applying signal detection theory to contingency assessment. Comparative Cognition \& Behavior Reviews, 4, 116-134. Retrieved from http://psyc.queensu.ca/ccbr/index.html.

Singer, M., Gagnon, N., \& Richards, E. (2002). Strategies of text retrieval: A criterion shift account. Canadian Journal of Experimental Psychology, 56, 41-57. 
Spellman, B. A. (1996a). Acting as intuitive scientists: Judgments are made while controlling for alternative potential causes. Psychological Science, 7, 337-342.

Spellman, B. A. (1996b). Conditionalizing causality. In D. R. Shanks, K. J. Holyoak, \& D. L. Medin (Eds.), The psychology of learning and motivation (Causal learning, Vol. 34, pp. 167-200). San Diego, CA: Academic Press.

Stout, S. C., \& Miller, R. R. (2007). Sometimes-competing retrieval (SOCR): A formalization of the comparator hypothesis. Psychological Review, 114, 759-783.

Stretch, V., \& Wixted, J. T. (1998). On the difference between strength-based and frequency-based mirror effects in recognition memory. Journal of Experimental Psychology: Learning, Memory, and Cognition, 24, 1379-1396.

Thomas, E. A. C. (1975). Criterion adjustment and probability matching. Perception \& Psychophysics, 18, 158-162.

Treisman, M. (1984a). Contingent aftereffects and situationally coded criteria. In J. Gibbon \& L. G. Allan (Eds.), Timing and time perception (Annals of the New York Academy of Sciences (Vol. 423, pp. 131-141). New York: New York Academy of Sciences.

Treisman, M. (1984b). A theory of criterion setting: An alternative to the attention band and response ratio hypotheses in magnitude estimation and cross-modality matching. Journal of Experimental psychology: General, 113, 443-463.
Treisman, M., \& Williams, T. C. (1984). A theory of criterion setting with an application to sequential dependencies. Psychological Review, 91, 68-111.

Verde, M. F., \& Rotello, C. M. (2007). Memory strength and the decision process in recognition memory. Memory \& Cognition, $35,254-262$.

\section{Author's note}

Samuel D. Hannah, Department of Psychology, Neuroscience, \& Behaviour, McMaster University, Hamilton, Ontario, L8S 4K1, Canada. Lorraine G. Allan, Department of Psychology, Neuroscience, \& Behaviour, McMaster University, Hamilton, Ontario, L8S 4K1, Canada.

Samuel Hannah is now at the School of Psychology, University of Queensland, St. Lucia, Queensland, 4072, Australia. We thank Matthew Crump, Douglas Mewhort, Ralph Miller, Shepard Siegel, and Steven Stout for insightful and useful comments. The work reported in this paper was supported by research grants from the National Sciences and Engineering Research Council of Canada to Lorraine Allan and Shepard Siegel. 